\title{
Graph-Theoretic Connectivity Control of Mobile Robot Networks
}

\author{
This paper develops an analysis for groups of vehicles connected by a \\ communication network; control laws are formulated to accomplish tasks \\ requiring rendezvous, and swarm in group formations.
}

By Michael M. Zavlanos, Member IEeE, Magnus B. Egerstedt, Senior Member IEEE, And

George J. Pappas, Fellow IEEE

ABSTRACT | In this paper, we provide a theoretical framework for controlling graph connectivity in mobile robot networks. We discuss proximity-based communication models composed of disk-based or uniformly-fading-signal-strength communication links. A graph-theoretic definition of connectivity is provided, as well as an equivalent definition based on algebraic graph theory, which employs the adjacency and Laplacian matrices of the graph and their spectral properties. Based on these results, we discuss centralized and distributed algorithms to maintain, increase, and control connectivity in mobile robot networks. The various approaches discussed in this paper range from convex optimization and subgradient-descent algorithms, for the maximization of the algebraic connectivity of the network, to potential fields and hybrid systems that maintain communication links or control the network topology in a least restrictive manner. Common to these approaches is the use of mobility to control the topology of the underlying communication network. We discuss applications of connectivity control to multirobot rendezvous, flocking and formation control, where so far, network connectivity has been considered an assumption.

Manuscript received May 13, 2010; revised October 14, 2010 and March 15, 2011; accepted May 8, 2011. Date of publication July 12, 2011; date of current version August 19, 2011. The work of M. M. Zavlanos and G. J. Pappas was supported by the ONR HUNT MURI and ARO SWARMS MURI projects. The work of M. B. Egerstedt was supported by the ONR HUNT MURI project.

M. M. Zavlanos is with the Department of Mechanical Engineering, Stevens Institute of Technology, Hoboken, NJ07030 USA (e-mail: michael.zavlanos@stevens.edu). M. B. Egerstedt is with the Department of Electrical and Computer Engineering, Georgia Institute of Technology, Atlanta, GA 30332 USA (e-mail:

magnus@ece.gatech.edu).

G. J. Pappas is with the Department of Electrical and Systems Engineering, University of Pennsylvania, Philadelphia, PA 19104 USA (e-mail: pappasg@seas.upenn.edu).

Digital Object Identifier: 10.1109/JPROC.2011.2157884
KEYWORDS | Algebraic graph theory; convex and subgradient optimization; graph connectivity; hybrid systems

\section{INTRODUCTION}

Mobile robot networks have recently emerged as an inexpensive and robust way of addressing a wide variety of tasks ranging from exploration, surveillance, and reconnaissance, to cooperative construction and manipulation. The success of these stories relies on efficient information exchange and coordination between the members of the team. In fact, recent work on distributed consensus and state agreement has strongly depended on multihop communication for convergence and performance guarantees [1]-[14].

Multihop communication in multirobot systems has typically relied on constructs from graph theory, with weighted proximity and disc-based graphs gaining the most popularity. Besides their simplicity, these models owe their popularity to their resemblance to radio signal strength models, where the signals attenuate with the distance [15]-[17]. In this context, multihop communication becomes equivalent to network connectivity, defined as the property of a graph to transmit information between any pair of its nodes.

Network connectivity has been widely studied in the area of wireless and ad hoc networks. Of great importance in this field is the power management of the nodes for optimal routing and lifetime of the network, while ensuring connectivity [18]-[23]. This research has given rise to connectivity or topology control algorithms that regulate the transmission power of the nodes and, therefore, their communication range. Approaches range from cone-based 


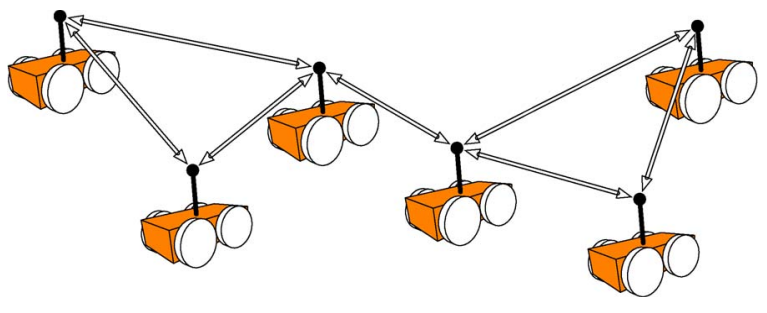

Fig. 1. Networks have long served as models of local interactions in the field mobile robotics. Robots are typically associated with the nodes of a graph and communication links with the edges.

[24]-[26] to distributed algorithms that do not involve any position information of the nodes [27], [28]. Related is also work on asymptotic bounds on the number of neighbors required to ensure connectivity in randomly deployed networks [29], as well as on the critical interference above which connectivity is lost [30]. However, this type of work focuses more on the power consumption and routing problem than the actuation and control.

Although networks have long served as models of local interactions in the field of mobile robotics (Fig. 1), until recently their structural properties have been assumed and decoupled from the control objectives, as in the case of connectivity in distributed consensus [1]-[14]. A first attempt to control the network structure was with the design of networks with maximal connectivity, where eigenvector structure-based approaches for tree networks [31], [32] were followed by optimization-based approaches applied to more general networks [33], [34]. These results were derived for static, state-independent, networks. Recently, controllability frameworks for state-dependent graphs were also proposed [35]. Nevertheless, the first work to treat connectivity as a control objective was [36], in the context of multirobot rendezvous. Since then, a large amount of research has been targeted in this direction, and a wide range of applications and solution techniques have been proposed.

A metric that is typically employed to capture connectivity of robotic networks is the second smallest eigenvalue $\lambda_{2}(\mathcal{L})$ of the Laplacian matrix $\mathcal{L}$ of the graph, also known as the algebraic connectivity or Fiedler value of the graph. It is well known that $\lambda_{2}(\mathcal{L})$ is a concave function of the Laplacian matrix, and when positive definite, it implies network connectivity [37]-[40]. This has given rise to optimization-based connectivity controllers that rely on maximization of the Fiedler value [41], [42]. Since $\lambda_{2}(\mathcal{L})$ is a function of the network's structure via the Laplacian matrix, connectivity algorithms that relied on it were initially centralized [41]. Only recently have there been subgradient algorithms for its distributed optimization [42]. Furthermore, the Fiedler value is a nondifferentiable function of the Laplacian matrix, which presents difficulties in designing feedback controllers to maintain it positive definite. Ways to overcome this problem involve either positive definiteness constraints on the determinant of the Laplacian matrix that is a differentiable function of the Laplacian [43], or distributed consensus on either Laplacian eigenvectors [44], [45] or on the network structure itself [46] for local estimation of the Fiedler value of the overall network.

Alternatively, connectivity can be captured by the sum of powers $\sum_{k=0}^{K} \mathcal{A}^{k}$ of the adjacency matrix $\mathcal{A}$ of the network, which represents the number of paths up to length $K$ between every pair of nodes in the graph [40]. By definition of graph connectivity, if this number is positive definite for $K=n-1$ and all pairs of nodes, then the network is connected ( $n$ denotes the number of nodes). For originally connected networks, maintaining positive definiteness of all positive entries of $\sum_{k=0}^{K} \mathcal{A}^{k}$ for any $K \leq n-1$, maintains paths of maximum length $K$ between agents and, as shown in [47], is sufficient to maintain connectivity of the network. This typically gives rise to optimization-based connectivity controllers [47], [48] that are often centralized due to the multihop agent dependencies that are introduced by the powers of the adjacency matrix. Since smaller powers correspond to shorter dependencies (paths), distribution is possible as $K$ decreases. If $K=1$, connectivity maintenance reduces to preserving the links of a connected spanning subgraph of the network and due to differentiability of the adjacency matrix, often results in feedback solution techniques. Discrete-time approaches are discussed in [36], [49], and [50], while [51]-[56] rely on local gradients that may also incorporate switching in the case of link additions. Switching between arbitrary spanning topologies has also been studied with the spanning subgraphs being updated by local auctions [46], distributed spanning tree algorithms [57], combination of information dissemination algorithms and graph picking games [58], or intermediate rendezvous [59], [60]. This class of approaches are typically hybrid, combining continuous link maintenance and discrete topology control. The algebraic connectivity $\lambda_{2}(\mathcal{L})$ and number of paths $\sum_{k=0}^{K} \mathcal{A}^{k}$ metrics can also be combined to give controllers that maintain connectivity, while enforcing desired multihop neighborhoods for all agents [61].

The results discussed above have been successfully applied to multiple scenarios that require network connectivity to achieve a global coordinated objective. Indicative of this work is recent literature on connectivity preserving rendezvous [36], [52], [56], [62], [63], flocking [55], [64], and formation control [56], [59], where so far connectivity had been an assumption. Further extensions and contributions involve connectivity control for double integrator agents [49], agents with bounded inputs [65]-[67] and indoor navigation [61], as well as for communication based on radio signal strength [68]-[71] and visibility constraints [36], [62], [72]-[74]. Periodic connectivity for robot teams that need to occasionally split in order to achieve individual objectives [75] and sufficient conditions for connectivity in leader-follower networks 
[76], also add to the list. Early experimental results have demonstrated efficiency of these algorithms also in practice [75], [77], [78].

In this paper, we focus on the works of [41]-[43], [46], [56], and [64], since they are the first to have formally addressed connectivity control of mobile networks for a wide range of applications and solution techniques. Our contribution is to present a cohesive overview of the key results in these papers in a unified framework. This includes basic notions of network connectivity and control-theoretic methods for connectivity guarantees and convergence. The results discussed in this work incorporate a variety of mathematical tools, ranging from spectral graph theory and semidefinite programming, to gradient-descent algorithms and hybrid systems. A byproduct of this work is to classify the available literature with respect to the connectivity metrics and solution techniques and provide a basis upon which future research can be built.

The rest of this paper is organized as follows. In Section II, we develop graph-theoretic models of communication and discuss network connectivity. In Section III, we present centralized [41] and distributed [42] optimization-based approaches to maximizing the algebraic connectivity of a network, while in Section IV, we discuss gradient-based feedback controllers that rely on the spectral properties of the network [43]. In Section V, we introduce distributed hybrid solutions to the problem [46], [56], while in Section IV, we discuss application of connectivity control to connectivity preserving rendezvous [56], flocking [64], and formation control [56].

\section{CONNECTIVITY IN MOBILE ROBOT NETWORKS}

Consider $n$ points robots in $\mathbf{R}^{d}$ and let $x_{i}(t) \in \mathbf{R}^{d}$ denote the position of robot $i$ at time $t \geq 0$. The robots can be described by either single integrator models

$$
\dot{x}_{i}(t)=u_{i}(t)
$$

where $u_{i}(t) \in \mathbf{R}^{d}$ denotes the control input to robot $i$ at time $t$, or double integrator models

$$
\begin{aligned}
& \dot{x}_{i}(t)=v_{i}(t) \\
& \dot{v}_{i}(t)=u_{i}(t)
\end{aligned}
$$

where $v_{i}(t) \in \mathbf{R}^{d}$ denotes the velocity of robot $i$ at time $t$. Assume further that the robots have integrated wireless communication capabilities and denote by $(i, j)$ a communication link between robots $i$ and $j$. With every communication link $(i, j)$, we associate a weight function

$$
w: \mathbf{R}^{d} \times \mathbf{R}^{d} \rightarrow \mathbf{R}_{+}
$$

such that

$$
w_{i j}(t)=w\left(x_{i}(t), x_{j}(t)\right)=f\left(\left\|x_{i j}(t)\right\|_{2}\right)
$$

for some $f: \mathbf{R}_{+} \rightarrow \mathbf{R}_{+}$, where $x_{i j}(t)=x_{i}(t)-x_{j}(t) .{ }^{1}$ We choose the function $f$ to be a decreasing function of the inter-robot distance $\left\|x_{i j}(t)\right\|_{2}$ such that

$$
1-\epsilon<f\left(\left\|x_{i j}(t)\right\|_{2}\right) \leq 1, \quad \text { if }\left\|x_{i j}(t)\right\|_{2}<\rho_{1}
$$

and

$$
0 \leq f\left(\left\|x_{i j}(t)\right\|_{2}\right)<\epsilon, \quad \text { if }\left\|x_{i j}(t)\right\|_{2}>\rho_{2}
$$

for $0<\rho_{1}<\rho_{2}$ and small enough $0<\epsilon<1$ (Fig. 2). This definition captures the fact that signal strength between wireless robots is strong up to a distance $\rho_{1}$ and then decreases rapidly until it practically vanishes beyond a distance $\rho_{2}$.

The system described above gives rise to a weighted state-dependent graph

$$
\mathrm{G}=(\mathbb{V}, \mathbb{W})
$$

where $\mathbb{V}=\{1, \ldots, n\}$ denotes the set of nodes indexed by the set of robots and $\mathbb{W}: \mathbb{V} \times \mathbb{V} \times \mathbf{R}_{+} \rightarrow \mathbf{R}_{+}$denotes the set of edge weights, such that

$$
\mathbb{W}(i, j, t)=w_{i j}(t)
$$

for $i, j \in \mathbb{V}$ and with $w_{i j}(t)$ as in (3). The set $\overrightarrow{\mathbb{E}}(t)=$ $\left\{(i, j) \mid w_{i j}(t)>0\right\}$ is called the set of directed edges of $\mathrm{G}$, while the unordered pair $\{i, j\}$ is an edge of $\mathrm{G}$ if $w_{i j}(t)>0$ or $w_{j i}(t)>0$. If $w_{i j}(t)=0$ implies $w_{j i}(t)=0$ for all $i, j \in \mathbb{V}$, then the weights are called weakly symmetric and the graph is called undirected. On the other hand, if $w_{i j}(t)=w_{j i}(t)$ for all $i, j \in \mathbb{V}$, then the weights are called symmetric. Clearly, if a graph has symmetric weights, then it is also undirected. Throughout this paper, we assume graphs $\mathrm{G}$ with symmetric weights that additionally have no loops, i.e., $w_{i i}(t)=0$ for all $i \in \mathbb{V}$. We also define the set of neighbors of node $i \in \mathbb{V}$ by $\mathbb{N}_{i}(t)=\{j \in \mathbb{V} \mid(i, j) \in \overrightarrow{\mathbb{E}}(t)\}$, which in the case of undirected graphs results in a mutual adjacency relationship between nodes, i.e., if $i \in \mathbb{N}_{j}(t)$ then $j \in \mathbb{N}_{i}(t)$. Similarly, we define a directed path of

${ }^{1}$ We denote by $\mathbf{R}_{+}$the set $[0, \infty)$ and by $\mathbf{R}_{++}$the set $(0, \infty)$. 


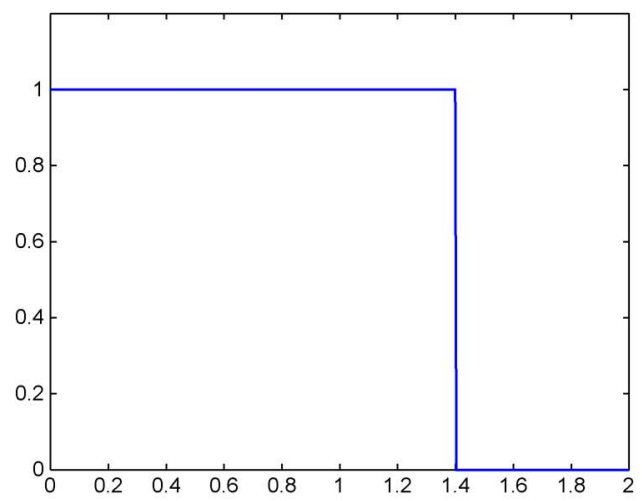

(a)

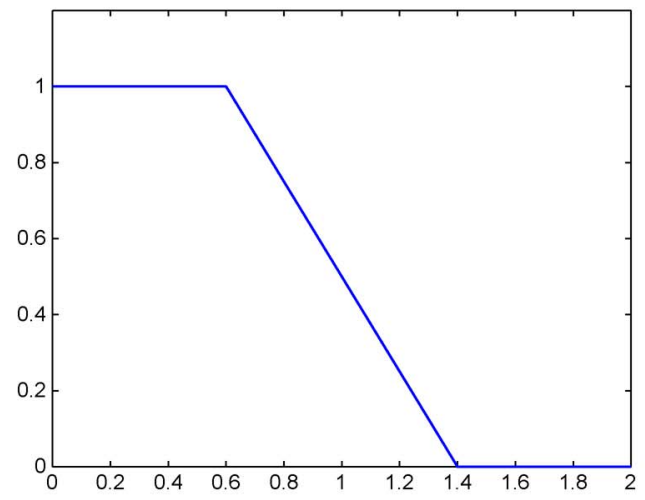

(c)

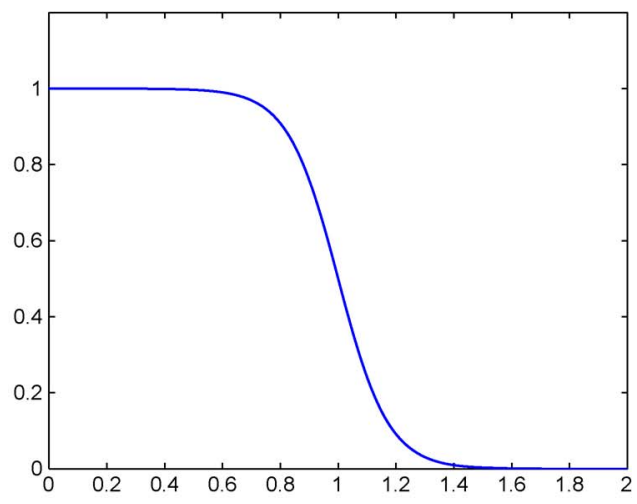

(b)

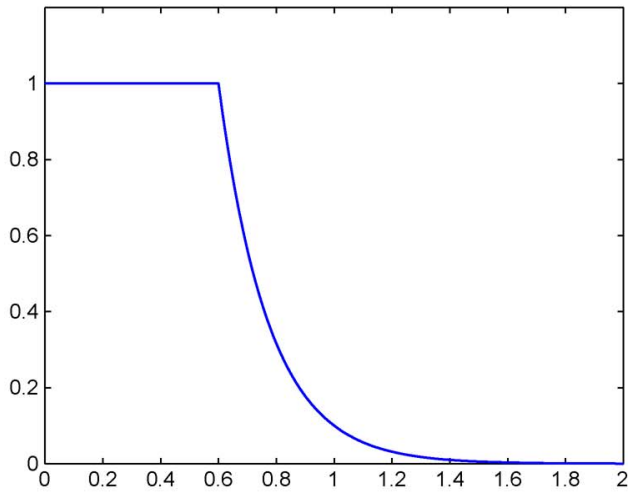

(d)

Fig. 2. Different choices for the function $f$. In particular, (a) $f(\boldsymbol{y})=\mathbf{1}$ if $y \leq \rho_{2}$; (b) $\boldsymbol{f}(\boldsymbol{y})=\mathbf{1} / \mathbf{1}+\boldsymbol{e}^{-\alpha(\boldsymbol{y}-\rho)}$ with $\alpha=\left(\mathbf{2} /\left(\rho_{2}-\rho_{1}\right)\right) \log ((\mathbf{1}-\epsilon) / \epsilon)$, and $\rho=\left(\rho_{1}+\rho_{2}\right) / \mathbf{2} ;$ (c) $f(\boldsymbol{y})=\left(\mathbf{1} /\left(\rho_{1}-\rho_{2}\right)\right) y-\left(\rho_{2} /\left(\rho_{1}-\rho_{2}\right)\right)$ if $\rho_{1} \leq \boldsymbol{y}<\rho_{2}$; and (d) $\boldsymbol{f}(\boldsymbol{y})=e^{-\alpha\left(\boldsymbol{y}-\rho_{1}\right)}$ if $\boldsymbol{y}>\rho_{1}$, with $\alpha=\left(\mathbf{1} /\left(\rho_{2}-\rho_{1}\right)\right) \log (\mathbf{1} / \epsilon)$. The above plots are for $\rho_{1}=0.6, \rho_{2}=1.4$, and $\epsilon=0.01$.

length $k$ by a sequence of $k+1$ distinct nodes $i_{0}, i_{1}, \ldots$, $i_{k} \in \mathbb{V}$ such that $\left(i_{p-1}, i_{p}\right) \in \overrightarrow{\mathbb{E}}(t)$ for all $1 \leq p \leq k$. If the graph $\mathrm{G}$ is undirected, then so are its paths. An important topological invariant of graphs is graph connectivity, which for the case of undirected graphs is defined as follows.

Definition 2.1 (Graph Connectivity): We say that an undirected graph $\mathrm{G} r$ is connected if for every pair of nodes there exists a path starting at one node and ending at the other.

Network connectivity is an important property of robotic networks designed to achieve global coordinated objectives, since it ensures information sharing via multihop communication paths between members of the team. This property can be efficiently captured using an equivalent algebraic representation of graphs by the adjacency and Laplacian matrices.

\section{A. Algebraic Definitions of Connectivity}

We define the adjacency matrix $\mathcal{A}(t) \in \mathbf{R}_{+}^{n \times n}$ of the weighted graph Gr with entries

$$
[\mathcal{A}(t)]_{i j}=w_{i j}(t)
$$

Clearly, if the network has symmetric weights, then the adjacency matrix is a symmetric matrix. Furthermore, if the weights satisfy $w_{i j}(t) \in\{0,1\}$ [Fig. 2(a)], then the powers of the adjacency matrix of a graph are closely related to network connectivity. In particular, we have the following result [40].

Theorem 2.2 (Graph Connectivity): The entry $\left[\mathcal{A}^{k}(t)\right]_{i j}$ of the matrix $\mathcal{A}^{k}(t)$ is the number of paths of length $k$ from node $i$ to node $j$ in Gr. Therefore, the graph $G$ is connected if and only if there exists an integer $K$ such that all the entries of the matrix $\mathcal{C}_{K}(t)=\sum_{k=0}^{K} \mathcal{A}^{k}(t)$ are nonzero.

Note that the integer $K$ in Theorem 2.2 is upper bounded by $n-1$, since this is the length of the longest possible path in a network of $n$ nodes. Note also that for any $K \leq n-1$ the inequality

$$
\left[\mathcal{C}_{K}(t)\right]_{i j}>0
$$

enforces paths of maximum length $K$ between nodes $i$ and $j$ in V. It is shown in [47] that, for initially connected 
networks, requiring that $\left[C_{K}(t)\right]_{i j}>0$, for any $K \leq n-1$, whenever $\left[C_{K}(0)\right]_{i j}>0$ is sufficient for network connectivity for all time $t \geq 0$. This result can be easily understood if applied for $K=1$, where it states that maintaining all one-hop links of an originally connected network is sufficient for connectivity for all time. In what follows, when relying on the matrix $\mathcal{C}_{K}(t)$ to ensure connectivity, we only consider the case $K=1$. The general case is discussed in [47] and [48].

Alternatively, graph connectivity can be captured using the Laplacian matrix $\mathcal{L}(t) \in \mathbf{R}^{n \times n}$ of the network Gr, which is defined by

$$
[\mathcal{L}(t)]_{i j}= \begin{cases}-w_{i j}(t), & \text { if } i \neq j \\ \sum_{s \neq i} w_{i s}(t), & \text { if } i=j .\end{cases}
$$

If $\mathcal{D}(t)=\operatorname{diag}\left(\sum_{j=1}^{n} w_{i j}(t)\right)$ denotes the diagonal matrix of degrees of the network, also called the Valency matrix of $\mathrm{G}$, then the Laplacian matrix can be written as

$$
\mathcal{L}(t)=\mathcal{D}(t)-\mathcal{A}(t)
$$

The Laplacian matrix of a network Gr with symmetric weights is always a symmetric positive-semidefinite matrix with spectral properties closely related to network connectivity, as it can be seen from the following theorem [40].

Theorem 2.3: Let

$$
0 \leq \lambda_{1}(\mathcal{L}(t)) \leq \lambda_{2}(\mathcal{L}(t)) \leq \cdot \leq \lambda_{n}(\mathcal{L}(t))
$$

be the ordered eigenvalues of the Laplacian matrix $\mathcal{L}(t)$. Then, $\lambda_{1}(\mathcal{L}(t))=0$ with corresponding eigenvector 1 , i.e., the $n \times 1$ vector of all entries equal to 1 . Moreover, $\lambda_{2}(\mathcal{L}(t))>0$ if and only if $\mathrm{G}$ is connected.

Besides an indicator of connectivity, the second smallest eigenvalue $\lambda_{2}(\mathcal{L}(t))$ of the Laplacian matrix of $\mathrm{G}$, also called the algebraic connectivity or Fiedler value of the network, is also a measure of the robustness of the network to link failures, captured by the notion of $k$-connectivity [40].

Definition 2.4 (k-Connectivity): Let $\eta(\mathrm{G})$ be the minimum number of edges that if removed from $G$ increase its number of connected components. Then, for any $k \leq \eta(\mathrm{G})$ the undirected graph $\mathrm{G}$ is called $k$-connected.

The edge connectivity $\eta(\mathrm{G})$ and algebraic connectivity $\lambda_{2}(\mathcal{L}(t))$ are related by the inequality [40]
Therefore, if $\lambda_{2}(\mathcal{L}(t))>k-1$, then the network $\mathrm{G}$ is $k$-connected. Note that if $k=1$, then $k$-connectivity reduces to the usual definition of connectivity (Definition 2.1). The results discussed above give rise to the following statement of the connectivity control problem.

Problem 1 (Network Connectivity Control): Given an initially connected state-dependent network $\mathrm{G}$, design distributed controllers $\left\{u_{i}(t)\right\}_{i=1}^{n}$ for the robots so that the closed-loop system (1) or (2) guarantees that $\mathrm{G}$ is $k$-connected for all time.

In what follows, we discuss optimization [41], [42] and feedback-based [43], [46], [56] solutions to Problem 1 that employ both connectivity metrics developed above, i.e., the adjacency matrix $\mathcal{A}(t)$ and its powers as well as the algebraic connectivity $\lambda_{2}(\mathcal{L}(t))$. We unify these approaches under a common control framework and characterize them with respect to the amount of distribution they possess.

\section{OPTIMIZATION-BASED CONNECTIVITY CONTROL}

Observe that $\lambda_{2}(\mathcal{L}(t))$ is a concave function of $\mathcal{L}(t)$ in the space $\mathbf{1}^{\perp}$ given by the infimum of a set of linear functions in $\mathcal{L}(t)$, i.e.,

$$
\lambda_{2}(\mathcal{L}(t)) z^{T} z \leq z^{T} \mathcal{L}(t) z
$$

for all $z \in \mathbf{1}^{\perp}$, or equivalently

$$
\lambda_{2}(\mathcal{L}(t))=\inf _{z \in \mathbf{1}^{\perp}} \frac{z^{T} \mathcal{L}(t) z}{z^{T} z} .
$$

Therefore, maximization of $\lambda_{2}(\mathcal{L}(t))$ gives rise to optimization-based approaches to the connectivity control problem. In other words, a sufficient solution to Problem 1 can be obtained by solving the optimization problem

$$
\max _{x \in \mathbf{R}^{\operatorname{dn}}} \lambda_{2}(\mathcal{L}(x))
$$

where $x=\left[\begin{array}{llll}x_{1} & x_{2} & \ldots & x_{n}\end{array}\right]^{T} \in \mathbf{R}^{d n}$ denotes the vector of all robot positions. The two approaches to this problem that we discuss rely on concavity of the state-independent problem

$$
\lambda_{2}(\mathcal{L}(t)) \leq \eta(\mathrm{G}) .
$$

$$
\max _{\mathcal{L} \in \mathbf{S}^{n}} \lambda_{2}(\mathcal{L})
$$


to obtain an equivalent convex formulation, and then propose centralized and distributed iterative algorithms, respectively, to introduce the nonconvex dependence on the state $x \in \mathbf{R}^{d n}$.

\section{A. Centralized Connectivity Maximization}

The key idea behind a centralized solution to problem (8) is to employ the following result that relates positive definiteness of the algebraic connectivity to positive definiteness of a quadratic expression of the Laplacian matrix [41].

Proposition 3.1: Let $\mathcal{P}=\left[\begin{array}{lll}p_{1} & \ldots & p_{n-1}\end{array}\right] \in \mathbf{R}^{n \times n-1}$ be such that $p_{i}^{T} \mathbf{1}=0$ for all $i=1, \ldots, n-1$ and $p_{i}^{T} p_{j}=0$ for all $i \neq j$. Then, $\lambda_{2}(\mathcal{L})>0$ if and only if $\mathcal{P}^{T} \mathcal{L P} \succ 0$.

Proof: Since for any graph we have that $\mathcal{L} \succeq 0$ and $\mathcal{L} \mathbf{1}=\mathbf{0}$, the smallest eigenvalue $\lambda_{1}(\mathcal{L})=0$ is always zero and $\operatorname{rank}(\mathcal{L}) \leq n-1$. This implies that $\lambda_{2}(\mathcal{L})>0$ if and only if $w^{T} \mathcal{L} w>0$ for all $w \in \mathbf{1}^{\perp}$.

Let $z \in \mathbf{R}^{n-1}$ and consider the quadratic form $z^{T} \mathcal{P}^{T} \mathcal{L} \mathcal{P} z=(\mathcal{P} z)^{T} \mathcal{L} \mathcal{P} z$. Let $w=\mathcal{P} z$. Since $\mathcal{P}$ is full rank and $\mathbf{1}^{T} w=\mathbf{1}^{T} \mathcal{P} z=0$ for any $z \in \mathbf{R}^{n-1}$, the mapping $w=P z$ defines an injection between $\mathbf{R}^{n-1}$ and $\mathbf{1}^{\perp}$. Therefore, $w^{T} \mathcal{L} w>0$ for all $w \in \mathbf{1}^{\perp}$ if and only if $z^{T} \mathcal{P}^{T} \mathcal{L} \mathcal{P} z>0$ for all $z \in \mathbf{R}^{n-1}$.

Proposition 3.1 results in an equivalent convex formulation for problem (8) by

$$
\begin{array}{ll}
\max _{\mathcal{L} \in \mathbf{S}^{n}} & \gamma \\
\text { s.t. } & \mathcal{P}^{T} \mathcal{L} \mathcal{P} \succ \gamma \mathcal{I}_{n-1}
\end{array}
$$

which can be solved for the optimal Laplacian matrix $\mathcal{L}_{\star}$ using readily available tools from semidefinite programming [79].

To obtain a set of trajectories that drive the robots from a set of initial configurations to a final configuration with associated Laplacian matrix $\mathcal{L}_{\star}$, Kim and Mesbahi [41] introduce state dependence of the network $\mathrm{G}$ via the set of edge weights described in Fig. 2(d). Along with a set of minimum distance constraints $\left\|x_{i j}\right\|_{2} \geq \rho_{1}$, this gives rise to the optimization problem

$$
\begin{array}{ll}
\max _{x \in \mathbf{R}^{\mathrm{d} n}} & \gamma \\
\text { s.t. } & \mathcal{P}^{T} \mathcal{L}(x) \mathcal{P} \succ \gamma \mathcal{I}_{n-1} \\
& \left\|x_{i j}\right\|_{2}^{2} \geq \rho_{1}^{2}
\end{array}
$$

for all $i<j$, which now assumes a nonconvex form. Solution of problem (10) for a trajectory $x(t) \in \mathbf{R}^{d n}$ is achieved by an iterative algorithm that maximizes the algebraic connectivity at every step. For this, the distances $\left\|x_{i j}\right\|_{2}^{2}$ are differentiated and then discretized by Euler's first-order method to give

$$
2\left(x_{i}^{s+1}-x_{j}^{s+1}\right)^{T}\left(x_{i}^{s}-x_{j}^{s}\right)=[\mathcal{X}]_{i j}^{s+1}-[\mathcal{X}]_{i j}^{s}
$$

where $\mathcal{X} \in \mathbf{R}_{+}^{n \times n}$ is a Euclidean distance matrix, such that $[\mathcal{X}]_{i j}=\left\|x_{i j}\right\|_{2}^{2}$ and $s$ denotes the iteration index. Similarly, differentiating and discretizing the weights $w_{i j}$ gives

$$
w_{i j}^{s+1}=w_{i j}^{s}+\left.\frac{\partial f\left([\mathcal{X}]_{i j}\right)}{\partial[\mathcal{X}]_{i j}}\right|_{s}\left([\mathcal{X}]_{i j}^{s+1}-[\mathcal{X}]_{i j}^{s}\right)
$$

which results in a discrete Laplacian matrix $\mathcal{L}\left(x^{s}\right)$. Substituting in problem (10) gives

$$
\begin{array}{cl}
\max _{x^{s+1} \in \mathbf{R}^{d n}} & \gamma \\
\text { s.t. } & \mathcal{P}^{T} \mathcal{L}\left(x^{s+1}\right) \mathcal{P} \succ \gamma \mathcal{I}_{n-1},[\mathcal{X}]_{i j}^{s+1} \geq \rho_{1}^{2} \\
& 2\left(x_{i}^{s+1}-x_{j}^{s+1}\right)^{T}\left(x_{i}^{s}-x_{j}^{s}\right) \\
& =[\mathcal{X}]_{i j}^{s+1}-[\mathcal{X}]_{i j}^{s}
\end{array}
$$

for all $i<j$. Problem (11) is essentially a linear approximation to problem (10) and, therefore, there is a potential for inconsistencies between the robot positions and their pairwise distances. This problem can be resolved if a Euclidean distance constraint is enforced on the matrix $\mathcal{X} \in \mathbf{R}_{+}^{n \times n}$. Such a constraint can take the form of a linear matrix inequality, which is due to the following result.

Theorem 3.2 (Euclidean Distance Matrix): A matrix $\mathcal{X} \in \mathbf{R}_{+}^{n \times n}$ is a Euclidean distance matrix if and only if $\mathcal{J X} \mathcal{J} \preceq 0$ and $[\mathcal{X}]_{i i}=0$ for all $i=1, \ldots, n$, where $\mathcal{J}=\mathcal{I}_{n}-11^{\mathrm{T}} / n$.

Therefore, including the Euclidean distance matrix constraints from Theorem 3.2 to the maximization problem (11) ensures that there are no inconsistencies between the robot positions and the inter-robot distances. The iterative greedy algorithm proposed by Kim and Mesbahi [41] is guaranteed to converge as the sequence of algebraic connectivities generated by it is nondecreasing and upper bounded by $n-1$.

\section{B. Distributed Connectivity Maximization}

A distributed solution to problem (8) can be obtained by supergradient optimization [42]. In particular, a 
supergradient matrix for $\lambda_{2}(\mathcal{L}(x))$ can be obtained by observing that

$$
\lambda_{2}(\tilde{\mathcal{L}}) z_{2}^{T} z_{2} \leq z_{2}^{T} \tilde{\mathcal{L}} z_{2}
$$

where $\tilde{\mathcal{L}} \neq \mathcal{L}$ and $z_{2} \in \mathbf{1}^{\perp}$ is the unit eigenvector of $\mathcal{L}$ corresponding to $\lambda_{2}(\mathcal{L})$. The right-hand side of $(12)$ can be further expanded to give

$$
\begin{aligned}
z_{2}^{T} \tilde{\mathcal{L}} z_{2} & =z_{2}^{T} \mathcal{L} z_{2}+z_{2}^{T}(\tilde{\mathcal{L}}-\mathcal{L}) z_{2} \\
& =\lambda_{2}(\mathcal{L})+\left\langle z_{2} z_{2}^{T},(\tilde{\mathcal{L}}-\mathcal{L})\right\rangle
\end{aligned}
$$

which substituted in (12) gives

$$
\lambda_{2}(\tilde{\mathcal{L}}) z_{2}^{T} z_{2} \leq \lambda_{2}(\mathcal{L})+\left\langle z_{2} z_{2}^{T},(\tilde{\mathcal{L}}-\mathcal{L})\right\rangle .
$$

Therefore, the matrix $\mathcal{G}=z_{2} z_{2}^{T}$ is a supergradient for $\lambda_{2}(\mathcal{L})$. Then, the optimal Laplacian matrix $\mathcal{L}_{\star}$ can be obtained as the limit of the subgradient iteration

$$
\mathcal{L}_{\star}^{s+1}=\mathcal{L}_{\star}^{s}+\alpha^{s} \mathcal{G}^{s} .
$$

If the step size $\alpha^{s}$ is the coefficient of a not summable but square summable series, then the supergradient method converges to the optimal value. Distributed computation of the supergradient $\mathcal{G}^{s}$ as well as of the eigenvectors of the Laplacian matrix $\mathcal{L}$ is discussed in [80]. According to this scheme, every robot $i$ computes its own row of the Laplacian matrix $\mathcal{L}_{\star}^{s}$, denoted by $\left[\mathcal{L}_{\star}^{s}\right]_{i}$.

To obtain a set of trajectories that drive the robots from an initial configuration to a final configuration associated with the optimal Laplacian matrix $\mathcal{L}_{\star}=\lim _{s \rightarrow \infty} \mathcal{L}_{\star}^{s}$, DeGennaro and Jadbabaie [42] propose a set of distributed motion controllers $\left\{u_{i}(t)\right\}_{i=1}^{n}$ for the robots that essentially track the sequence of Laplacians $\mathcal{L}_{\star}^{s}$ generated by the supergradient algorithm (13). State dependence of the network $\mathrm{G}$ is introduced via a set of symmetric weights that are according to Fig. 2(d) and give rise to a statedependent Laplacian matrix $\mathcal{L}(x)$ defined by (5). Therefore, associated with every iteration of the supergradient iteration algorithm (13) is a motion control stage, which for every robot $i$ is captured by the following optimization problem:

$$
\min _{x_{i} \in \mathbf{R}^{d}}\left\|[\mathcal{L}(x)]_{i}-\left[\mathcal{L}_{\star}^{s}\right]_{i}\right\|_{2}^{2}
$$

where $[\mathcal{L}(x)]_{i}$ denotes the $i$ th row of the Laplacian matrix as a function of the robots' positions, and $\left[\mathcal{L}_{\star}^{\mathcal{s}}\right]_{i}$ is the ith row of the optimal Laplacian computed by robot $i$ at the sth step of the supergradient iteration. The above optimization problem is solved using potential functions and results in a controller

$$
u_{i}(t)=-\sum_{j \in \mathbb{N}_{i}} \nabla_{x_{i}} V_{i j}(t)
$$

for every robot $i$, where

$$
V_{i j}(t)= \begin{cases}\left(\left\|x_{i j}\right\|_{2}^{2}-\left[\mathcal{L}_{\star}^{s}\right]_{i j}^{-1}\right)^{2}, & \text { if }\left\|x_{i j}\right\|_{2} \leq \rho_{2} \\ \left(\rho_{2}-\left[\mathcal{L}_{\star}^{s}\right]_{i j}^{-1}\right)^{2}, & \text { if }\left\|x_{i j}\right\|_{2}>\rho_{2}\end{cases}
$$

and $\left[\mathcal{L}_{\star}^{s}\right]_{i j}^{-1}$ is the desired distance between robots $i$ and $j$, given by the inverse of the $(i, j)$ th entry of the target Laplacian matrix. It is shown in [42] that under certain boundedness conditions on the tracking error associated with the optimal Laplacian $\mathcal{L}_{\star}^{s}$, the supergradient algorithm converges.

\section{CONTINUOUS FEEDBACK CONNECTIVITY CONTROL}

Both approaches discussed in Section III employ discrete iterative algorithms to control the nondifferentiable algebraic connectivity $\lambda_{2}(\mathcal{L}(x))$. However, Proposition 3.1 and the fact that the determinant of any matrix is equal to the product of its eigenvalues gives that $\lambda_{2}(\mathcal{L}(x))>0$ if and only if $\operatorname{det}\left(\mathcal{P}^{T} \mathcal{L}(x) \mathcal{P}\right)>0$. Using this observation for state-dependent networks $G$ with edge weights as in Fig. 2(b), Zavlanos and Pappas [43] propose a class of potential fields $\phi: \mathbf{R}^{d n} \rightarrow \mathbf{R}_{+}$that treat connectivity violation as an obstacle in the configuration space. This is captured in the following result.

Proposition 4.1: Define the potential function

$$
\phi(x)=\log \operatorname{det}\left(\mathcal{P}^{T} \mathcal{L}(x) \mathcal{P}\right)^{-1} .
$$

Then, the closed-loop system (1) with $u=-\nabla_{x} \phi(x)$ guarantees that $\mathrm{G}$ is connected for all time.

Proof: The proof of this result relies on positive invariance of the level sets $\phi^{-1}([0, c])=\left\{x \in \mathbf{R}^{d n} \mid \phi(x) \leq\right.$ c) of $\phi$, which is due to the fact that $\dot{\phi}(x)=$ $-\left\|\nabla_{x} \phi(x)\right\|_{2}^{2} \leq 0$.

Note that the potential $\phi$ is a convex function of the Laplacian matrix $\mathcal{L}$ [79]. However, dependence of the Laplacian on the state via the edge weights makes $\phi$ a nonconvex function of the $x \in \mathbf{R}^{d n}$. Therefore, even though $\operatorname{det}\left(\mathcal{P}^{T} \mathcal{L}(x) \mathcal{P}\right)$ increases as a result of 
Proposition 4.1, $\lambda_{2}(\mathcal{L}(x))$ might actually decrease. This implies that the proposed control scheme ensures only local maximization of $\lambda_{2}(\mathcal{L}(x))$. Zavlanos and Pappas [43] conclude by providing a closed-form expression for the controller in Proposition 4.1.

Proposition 4.2: The controller $u=-\nabla_{x} \phi(x)$ is given by

$$
u=\frac{1}{\operatorname{det} \mathcal{M}(x)}\left[\begin{array}{c}
\operatorname{tr}\left[\mathcal{M}^{-1}(x) \frac{\partial}{\partial x_{1}} \mathcal{M}(x)\right] \\
\vdots \\
\operatorname{tr}\left[\mathcal{M}^{-1}(x) \frac{\partial}{\partial x_{n}} \mathcal{M}(x)\right]
\end{array}\right]
$$

where $\mathcal{M}(x)=\mathcal{P}^{T} \mathcal{L}(x) \mathcal{P}$.

Proof: Let $\mathcal{M}(x)=\mathcal{P}^{T} \mathcal{L}(x) \mathcal{P}$ and denote by $c_{i j}(x)$ the cofactor of the entry $m_{i j}(x)$ of the matrix $\mathcal{M}(x)$. Let $\mathcal{C}(x)$ denote the cofactor matrix and denote by $c_{i j}^{T}(x)$ the $(i, j)$ th entry of $\mathcal{C}^{T}(x)$, i.e., $c_{i j}^{T}(x)=c_{j i}(x)$. Since the determinant is a differentiable function of matrix entries, in particular, it is a sum of products of entries, the chain rule gives

$$
\frac{\partial}{\partial x_{k}} \operatorname{det} \mathcal{M}(x)=\sum_{i=1}^{n-1} \sum_{j=1}^{n-1}\left(\frac{\partial}{\partial m_{i j}} \operatorname{det} \mathcal{M}(x)\right) \frac{\partial}{\partial x_{k}} m_{i j}(x)
$$

For all $j=1, \ldots, n-1$, computation of the Laplace expansion of the determinant along the $j$ th column gives $\operatorname{det} \mathcal{M}(x)=\sum_{i=1}^{n-1} c_{i j}(x) m_{i j}(x)$ and hence $\left(\partial / \partial m_{i j}\right)$ $\operatorname{det} \mathcal{M}(x)=c_{i j}(x)$. Therefore

$$
\begin{aligned}
\frac{\partial}{\partial x_{k}} \operatorname{det} \mathcal{M}(x) & =\sum_{i=1}^{n-1} \sum_{j=1}^{n-1} c_{i j}(x) \frac{\partial}{\partial x_{k}} m_{i j}(x) \\
& =\sum_{j=1}^{n-1} \sum_{i=1}^{n-1} c_{j i}^{T}(x) \frac{\partial}{\partial x_{k}} m_{i j}(x) \\
& =\operatorname{tr}\left[\mathcal{C}^{T}(x) \frac{\partial}{\partial x_{k}} \mathcal{M}(x)\right] .
\end{aligned}
$$

A direct consequence of the Laplace expansion of the determinant is the identity $I \cdot \operatorname{det} \mathcal{M}(x)=\mathcal{M}(x) \mathcal{C}^{T}(x)$. Proposition 4.1 guarantees that $\lambda_{2}(\mathcal{L}(x))>0$ for all time, and so $\operatorname{det} \mathcal{M}(x)>0$ for all $x \in \mathbf{R}^{d n}$. Thus, $\mathcal{M}(x)$ is always positive definite, and hence invertible. Therefore, by left multiplication of the previous identity by $\mathcal{M}^{-1}(x)$, we get $\mathcal{M}^{-1}(x) \cdot \operatorname{det} \mathcal{M}(x)=\mathcal{C}^{T}(x)$, and substituting in the expression for $\left(\partial / \partial x_{k}\right) \operatorname{det} \mathcal{M}(x)$, we get

$$
\begin{aligned}
\frac{\partial}{\partial x_{k}} \operatorname{det} \mathcal{M}(x) & =\operatorname{tr}\left[\operatorname{det} \mathcal{M}(x) \cdot \mathcal{M}^{-1}(x) \frac{\partial}{\partial x_{k}} \mathcal{M}(x)\right] \\
& =\operatorname{det} \mathcal{M}(x) \cdot \operatorname{tr}\left[\mathcal{M}^{-1}(x) \frac{\partial}{\partial x_{k}} \mathcal{M}(x)\right]
\end{aligned}
$$

where $\mathcal{M}^{-1}(x)=\left(\mathcal{P}^{T} \mathcal{L}(x) \mathcal{P}\right)^{-1}$ and $\left(\partial / \partial x_{k}\right) \mathcal{M}(x)=$ $\mathcal{P}^{T}\left(\partial / \partial x_{k}\right) \mathcal{L}(x) \mathcal{P}$, and the result follows directly from Proposition 4.1 and a simple application of the chain rule.

\section{HYBRID FEEDBACK CONNECTIVITY CONTROL}

The approach discussed in Section IV is centralized since every robot requires knowledge of the whole network structure captured by $\mathcal{L}(x)$ to compute its controller (Proposition 4.2). The key idea employed in [56] and [46] to regulate the structure of the proximity-based network $G$ in a distributed fashion is the introduction of a binary control signal $\sigma \in\{0,1\}^{n \times n}$, such that

$$
[\sigma]_{i j}= \begin{cases}1, & \text { to activate the } \operatorname{link}(i, j) \in \overrightarrow{\mathbb{E}} \\ 0, & \text { to deactivate the } \operatorname{link}(i, j) \in \overrightarrow{\mathbb{E}}\end{cases}
$$

This gives rise to the weighted graph $\mathrm{G}_{\sigma}=\left(\mathbb{V}, \mathbb{W}_{\sigma}\right)$ where $\mathbb{W}_{\sigma}: \mathbb{V} \times \mathbb{V} \times \mathbf{R}_{+} \rightarrow \mathbf{R}_{+}$is the set of edge weights such that

$$
W_{\sigma}(i, j, t)=w_{i j}^{\sigma}(t)
$$

with $w_{i j}^{\sigma}=w_{i j}[\sigma]_{i j}$, for $w_{i j}$ given by (3). Therefore, the control signal $\sigma$ is essentially a discrete switch on the links of the network $G$, but only affects existing links for which $w_{i j}>0$. The edge and neighbor sets associated with the graph $\mathbb{G}_{\sigma}$ are defined by $\overrightarrow{\mathbb{E}}_{\sigma}=\left\{(i, j) \mid w_{i j}^{\sigma}>0\right\}$ and $\mathbb{N}_{i}^{\sigma}=$ $\left\{j \in \mathbb{V} \mid(i, j) \in \overrightarrow{\mathbb{E}}_{\sigma}\right\}$, respectively. Based on this idea, Ji and Egerstedt [56] and Zavlanos and Pappas [46] propose a hybrid model for the mobile network G consisting of single integrator robots (1) and controllers given by

$$
u_{i}^{\sigma}=-\sum_{j \in \mathbb{N}_{i}^{\sigma}} \nabla_{x_{i}} \psi_{i j}
$$

The functions $\psi_{i j}: \mathbf{R}_{+} \rightarrow \mathbf{R}_{+}$are artificial potential functions defined on the links of the network, which in the case of connectivity control take the form (Fig. 3)

$$
\psi_{i j}=\frac{1}{\rho_{2}^{2}-\left\|x_{i j}\right\|_{2}^{2}}
$$

to ensure link preservation between adjacent robots. The rest of this section discusses two particular choices for the control signal $\sigma$ that ensure connectivity of the mobile network G. 


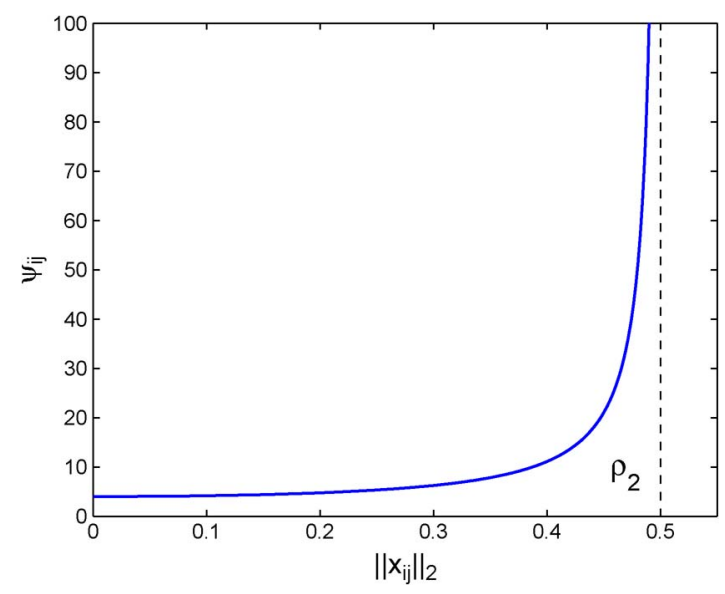

Fig. 3. The artificial potential function $\psi_{i j}\left(\left\|x_{i j}\right\|_{2}\right)$. The function is symmetric with respect to $x_{i}$ and $x_{j}$, and when bounded, it guarantees edge preservation for $\left\|x_{i j}\right\|_{2} \rightarrow \rho_{2}$. Here, the function is plotted for $\rho_{2}=\boldsymbol{0} .5$.

\section{A. Maintaining Communication Links}

The approach followed in [56] relies on maintaining and increasing the number of links in the network. Since

$$
\lim _{\left\|x_{i j}\right\|_{2} \rightarrow \rho_{2}^{-}} \psi_{i j}=\infty
$$

infinite energies $\psi_{i j}$ take place in the control laws (17) when two robots $i$ and $j$ form an edge between them, i.e., when they move within distance $\rho_{2}$ of each other. To address this problem, Ji and Egerstedt [56] introduce a hysteresis into the system through the signal $\sigma$ given by the state machine in Fig. 4. In particular, the signal $[\sigma]_{i j}$ is such that the total energy is affected by an edge $(i, j)$ that was previously not contributing to the total energy only when $\left\|x_{i j}\right\|_{2}<\rho_{1}$, where $0<\rho_{1}<\rho_{2}$ is the predefined switching threshold that regulates how fast inter-robot information is included in the control law. Once the edge is allowed to contribute to the total energy, it keeps doing

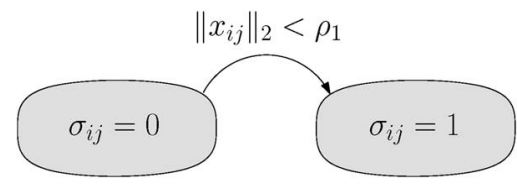

Fig. 4. Hysteresis protocol for adding interagent energy functions to the total energy function only when agents get within a distance $\rho_{1}$ of each other, rather than when they first encounter each other at a distance $\rho_{2}$. so for all subsequent times. In particular, the signal $[\sigma]_{i j}$ is defined by

$$
[\sigma]_{i j}\left(t^{+}\right)= \begin{cases}0, & \text { if }[\sigma]_{i j}\left(t^{-}\right)=0 \text { and }\left\|x_{i j}\right\|_{2} \geq \rho_{1} \\ 1, & \text { otherwise }\end{cases}
$$

where the notation $[\sigma]_{i j}\left(t^{+}\right)$and $[\sigma]_{i j}\left(t^{-}\right)$denotes the value of $[\sigma]_{i j}$ before and after the state transition in Fig. 4. It can be shown that this control scheme maintains all links in $\mathrm{G}_{\sigma}$ and, therefore, ensures connectivity of the network [46], [56].

Proposition 5.1: Consider the closed-loop system (1)(17). Then, all links in $\mathbb{G}_{\sigma}$ are maintained. Proof: Let

$$
\psi_{\sigma}=\frac{1}{2} \sum_{i=1}^{n} \psi_{i}^{\sigma}
$$

where $\psi_{i}^{\sigma}=\sum_{j \in \mathbb{N}_{i}^{\sigma}} \psi_{i j}$, denote the total energy of the system and observe that

$$
\begin{aligned}
\frac{1}{2} \sum_{i=1}^{n} \dot{\psi}_{i}^{\sigma} & =\frac{1}{2} \sum_{i=1}^{n} \sum_{j \in \mathbb{N}_{i}^{\sigma}} \dot{x}_{i j}^{T} \nabla_{x_{i j}} \psi_{i j} \\
& =\frac{1}{2} \sum_{i=1}^{n} \sum_{j \in \mathbb{N}_{i}^{\sigma}}\left(\dot{x}_{i}^{T} \nabla_{x_{i j}} \psi_{i j}-\dot{x}_{j}^{T} \nabla_{x_{i j}} \psi_{i j}\right) \\
& =\frac{1}{2} \sum_{i=1}^{n} \sum_{j \in \mathbb{N}_{i}^{\sigma}}\left(\dot{x}_{i}^{T} \nabla_{x_{i}} \psi_{i j}+\dot{x}_{j}^{T} \nabla_{x_{j}} \psi_{i j}\right) \\
& =\sum_{i=1}^{n} \sum_{j \in \mathbb{N}_{i}^{\sigma}} \dot{x}_{i}^{T} \nabla_{x_{i}} \psi_{i j}=\sum_{i=1}^{n} \dot{x}_{i}^{T} \nabla_{x_{i}} \psi_{i}^{\sigma}
\end{aligned}
$$

by symmetry of the functions $\psi_{i j}$. Therefore

$$
\dot{\psi}_{\sigma}=-\sum_{i=1}^{n}\left\|\nabla_{x_{i}} \psi_{i}^{\sigma}\right\|_{2}^{2} \leq 0
$$

which implies that the level sets $\psi_{\sigma}^{-1}([0, c])$ of $\psi_{\sigma}$ are positively invariant and, hence, no links are lost.

\section{B. Incorporating Link Deletions}

The approach followed in [46] extends the hysteresis model for link activations introduced in [56] to also account for connectivity preserving link deactivations. For this, Zavlanos and Pappas [46] propose a set of control signals $\left\{\sigma_{i}\right\}_{i=1}^{n}$, where $\sigma_{i} \in\{0,1\}^{n \times n}$ denotes the signal 


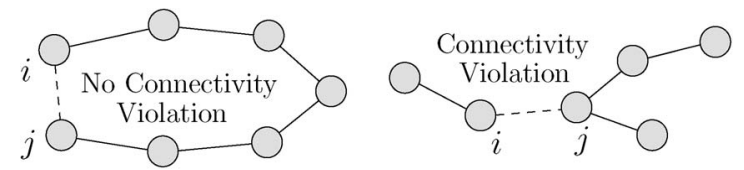

Fig. 5. Control challenges requiring knowledge of the network structure. Without such knowledge, deletion of a link $(i, j)$ can either violate connectivity (right) or not (left).

associated with robot $i$, that give rise to local neighbor sets $\mathbb{N}_{i}^{\sigma_{i}}$ defined as in Section V.

The key idea behind the approach developed in [46] is to employ distributed consensus to populate the signals $\sigma_{i}$ with nonadjacent active links and then use these signals to check link deactivations with respect to connectivity (Fig. 5). The latter objective is possible since connectivity verification does not require the actual edge weights, but only knowledge of what links in the network are active, which is captured by the signals $\sigma_{i}$. In other words, the signals $\sigma_{i}$ can be thought of as an abstraction of the adjacency matrices of the graphs $\mathrm{G}_{\sigma_{i}}$ obtained when the signals $\sigma_{i}$ are applied to Gr. The proposed update rule is ${ }^{2}$

$$
\sigma_{i}(s+1)=\neg\left(\sigma_{i}(s) \leftrightarrow \omega_{i}(s)\right)
$$

where $\omega_{i} \in\{0,1\}^{n \times n}$ is such that $\left[\omega_{i}\right]_{j k}=1$ if a control action is taken to activate or deactivate link $(j, k)$ (Table 1$)$. It is shown in [46] that $\omega_{i}$ can be decomposed into two disjoint components $\omega_{i}^{a}$ and $\omega_{i}^{d}$ regulating activations and deactivations, respectively, as

$$
\omega_{i}=\left((\underbrace{\left(\neg \sigma_{i} \wedge\left(\vee_{j \in \mathbb{N}_{i}^{\sigma_{i}}} \sigma_{j}\right)\right)}_{\mathrm{I}} \vee \underbrace{\left(\neg \sigma_{i} \wedge \eta_{i}\right)}_{\mathrm{II}}) \wedge \omega_{i}^{a}\right)_{\vee\left(\sigma_{i} \wedge \omega_{i}^{d}\right)}
$$

where

- the $(k, l)$ th entry of Term I (with $k, l \neq i$ ) is equal to 1 if there exists an active link between robots $k$ and $l$ that is known to robot $i$ 's neighbors, i.e., $\left[\mathrm{V}_{j \in \mathbb{N}_{i}^{\sigma_{i}}} \sigma_{j}\right]_{k l}=1$, but is not known to robot $i$, i.e., $\left[\neg \sigma_{i}\right]_{k l}^{i}=1$;

- the $(k, l)$ th entry of Term II with $k=i$ or $l=i$ is equal to 1 if there does not exist an active link between robots $k$ and $l$, i.e., $\left[\neg \sigma_{i}\right]_{k l}=1$, and is always zero if $k, l \neq i$.

\footnotetext{
${ }^{2}$ The symbols $\neg, \wedge, \vee, \rightarrow$, and $\leftrightarrow$ stand for the boolean operators NOT, AND, OR, IF, THEN, and IF AND ONLY IF, respectively (in the case of matrices, they are applied elementwise on their entries). The discrete time semantics in (19) are associated with discrete communication instances between adjacent robots.
}

Table 1 Link Dynamics

\begin{tabular}{|cc|c|}
\hline$\left[\sigma_{i}(s)\right]_{j k}$ & {$\left[\omega_{i}(s)\right]_{j k}$} & {$\left[\sigma_{i}(s+1)\right]_{j k}$} \\
\hline \hline 1 & 1 & 0 \\
1 & 0 & 1 \\
0 & 1 & 1 \\
0 & 0 & 0 \\
\hline \multicolumn{3}{|c}{} \\
\hline
\end{tabular}

The condition that $k=i$ or $l=i$ in Term II is captured by the matrix $\eta_{i}=\vee_{j \neq i}\left(e_{i} e_{j}^{T} \vee e_{j} e_{i}^{T}\right)$, where $e_{i}$ is an $n \times 1$ column vector with all entries 0 except for the ith entry that is 1 . Clearly, if the $(k, l)$ th entry of either Term I or Term II is equal to 1 , then this entry indicates a link that can possibly become activated if the activation control action becomes $\left[\omega_{i}^{a}\right]_{k l}=1$. Similarly, the control action $\omega_{i}^{d}$ can only deactivate links $(k, l)$ that robot $i$ considers active, i.e., $\left[\sigma_{i}\right]_{k l}=1$. It is shown in [46] that the dynamics (19) resemble a consensus algorithm with inputs on the control signals $\sigma_{i}$ that in the case of no inputs, i.e., if $\omega_{i}^{a}=1_{n \times n}$ and $\omega_{i}^{d}=0_{n \times n}$, reduce to the usual consensus update

$$
\sigma_{i}:=\vee_{j \in \mathbb{N}_{i}^{\sigma_{i}}}\left(\sigma_{i} \vee \sigma_{j}\right)
$$

as desired. The choice of the control actions $\omega_{i}^{a}$ and $\omega_{i}^{d}$ needs to satisfy the following two conditions:

1) $\sigma_{i}$ is updated with all active links in $\mathrm{G}_{\sigma}$;

2) connectivity of $\mathrm{G}_{\sigma}$ is not violated by link deactivations.

Condition 1) is satisfied by the link addition controller

$$
\omega_{i}^{a}=\underbrace{\left(\neg \eta_{i}\right)}_{\mathrm{III}} \vee \underbrace{\left(\eta_{\mathrm{i}} \wedge\left(\mathcal{X}<\frac{\rho_{1}^{2}}{n} \mathbf{1 1}^{T}\right)\right)}_{\mathrm{IV}},
$$

where

- Term III ensures that $\left[\omega_{i}^{a}\right]_{k l}=1$ whenever $k, l \neq i$, i.e., that all active links in the network known to robot $i$ 's neighbors will be activated in $\sigma_{i}$ as well;

- Term IV ensures that $\left[\omega_{i}^{a}\right]_{k l}=1$ whenever $k=i$ or $l=i$ and the distance $[\mathcal{X}]_{k l}=\left\|x_{k l}\right\|_{2}^{2}$ between robots $k$ and $l$ (with $k=i$ or $l=i$ ) is lower than the link activation threshold $\rho_{1}$, i.e., that links between robot $i$ and close-by agents will be activated ( $\mathcal{X} \in \mathbf{R}_{+}^{n \times n}$ denotes a Euclidean distance matrix).

Condition 2) needs to address the fact that simultaneous link deactivations by multiple nonadjacent robots may disconnect $\mathrm{G}_{\sigma}$ (Fig. 6). For this, Zavlanos and Pappas [46] propose a market-based framework to achieve agreement of all robots on one single link deactivation as the outcome of every auction. In particular, every robot $i$ 


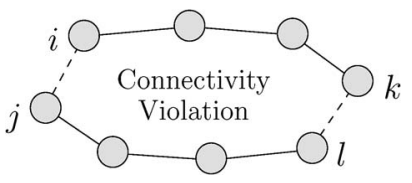

Fig. 6. Control challenges due to multiple link deletions. In the absence of an agreement protocol, simultaneous deletion of links $(i, j)$ and $(k, l)$ violates connectivity.

selects a neighbor $j$ in the set

$$
\mathbb{S}_{i}=\left\{k \in \mathbb{N}_{i}^{\sigma_{i}} \mid\left\|x_{i k}\right\|_{2} \in\left[\rho_{1}, \rho_{2}\right), \lambda_{2}\left(\mathcal{L}_{i}^{-k}\right)>0\right\}
$$

where $\mathcal{L}_{i}^{-k}$ is the Laplacian matrix of the network $\mathrm{G}_{\sigma_{i}}$ minus the link $(i, k)$, such that if the link $(i, j)$ with $j \in \mathbb{S}_{i}$ is deactivated, then the network $\mathrm{G}_{\sigma_{i}}$ remains connected. The rest of the algorithm relies on multihop propagation of

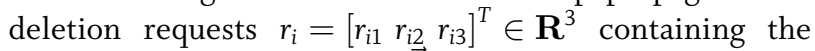
requested link $\left(r_{i 1}, r_{i 2}\right) \in \overrightarrow{\mathbb{E}}$ and an associated bid $r_{i 3} \in \mathbf{R}_{+}$, such that initially $r_{i 1}=i$ and $r_{i 2}=j \in \mathbb{S}_{i}$ for all robots $i$. With every communication round, request $r_{i}$ is updated with the request $r_{j}$ corresponding to the robot $j$ that has placed the highest bid $r_{j 3}$, i.e.,

$$
r_{i}=r_{j} \quad \text { with } \quad j \in \arg \max _{k \in \mathbb{N}_{i}^{\sigma_{i}}}\left\{r_{i 3}, r_{k 3}\right\}
$$

and employs a "maximum label" rule to break ties. Note that (21) is essentially a maximum consensus update on the bids $r_{i 3}$ and will converge to a common outcome $r_{i}$ for all robots when all bids have been compared to each other. If at least one robot has placed a positive bid, i.e., if $r_{i 3}>0$, then the controller

$$
\omega_{i}^{d}=e_{r_{i 1}} e_{r_{i 2}}^{T} \vee e_{r_{i 2}} e_{r_{i 1}}^{T},
$$

deactivates the link $\left(r_{i 1}, r_{i 2}\right)$ from $\mathrm{G}_{\sigma_{i}}$, and the process is repeated for a new link deactivation (Fig. 7). If $r_{i 3}=0$, then $\omega_{i}^{d}=0_{n \times n}$, i.e., no link is deactivated form $\mathrm{G}_{\sigma_{i}}$.

Communication time delays, packet losses, and the asymmetric network structure may result in auctions starting asynchronously, outdated information being used for future decisions, and consequently, robots reaching different decisions for the same auction. In the absence of a common global clock, Zavlanos and Pappas [46] propose an event-triggered synchronization scheme, where a triggering event corresponds to receipt of a communication message, which ensures that "fast" robots wait for their "slower" peers to reach a decision as well. Altogether, this framework gives rise to the following result.
Theorem 5.2 (Connectivity Maintenance): Assume that the network $\mathrm{G}_{\sigma}$ is initially connected. Then, the closedloop system (19), (20), (22) guarantees that $\mathrm{G}_{\sigma}$ remains connected for all time.

Proof: Assume that the local networks $\mathrm{G}_{\sigma_{i}}$ are initialized with nearest neighbor links only. Then, the proof relies on the following observations.

1) All network estimates $G_{\sigma_{i}}$ are spanning subgraphs of the overall network $\mathrm{G}_{\sigma}$, which implies that connectivity can be checked locally for $\mathrm{G}_{\sigma_{i}}$ and then extended to $\mathrm{G}_{\sigma}$.

2) The market-based maximum consensus (21) ensures agreement of all robots on the deactivation request which, therefore, does not violate connectivity.

3) Synchronization ensures that no outdated information is used in (21).

Consequently, links can be deactivated continuously one by one, without violating connectivity of the network.

\section{APPLICATIONS OF CONNECTIVITY CONTROL}

\section{A. Connectivity Preserving Rendezvous}

A canonical example in which connectivity maintenance is crucial is the so-called rendezvous problem. Here, the robots are required to meet at a common, not a priori

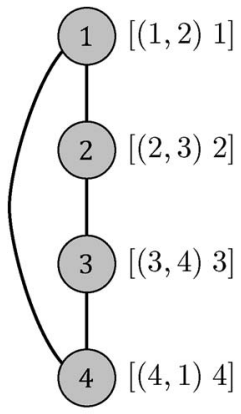

Initialization

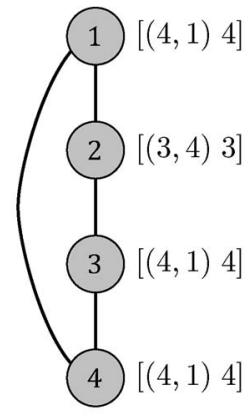

1st Communication Round

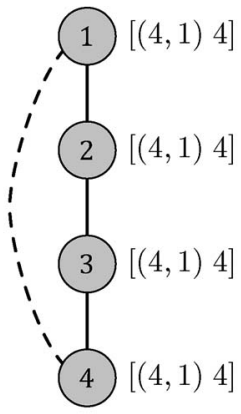

2nd Communication Round
Fig. 7. An example of a link deactivation auction taking place in a network of four robots. Next to every robot in brackets is shown its deletion request $r_{i}=\left[(i, j) b_{i}\right]$ containing a desired link $(i, j)$ with $j \in S_{i}$ that if deactivated does not violate connectivity, and the associated bid $b_{i}$. Initialization is as shown in the network at the left. During the first communication round, robot 1 compares its bid $b_{1}=1$ with the bids of its neighbors $b_{4}=4$ and $b_{2}=2$. Since among its neighbors, robot 4 has placed the highest bid, robot 1 updates its request with the request of robot 4 , i.e., $r_{1}=[(4,1) 4]$ [cf. (21)]. similar updates take place for the requests of the other robots. After two communication rounds, all robots have agreed on the request with the highest bid $[(4,1) 4]$. Then, robot 4 physically deactivates (dashed line) the link $(4,1)$ and along with all other robots updates its signal $\sigma_{i}$ [cf. (22)]. 
specified location without relying on global positioning. Instead, the only information available to them is the relative displacement, i.e., robot $i$, at position $x_{i}$, has access to $x_{j}-x_{i}$ if $i$ and $j$ are neighbors, i.e., if they are within sensing range of each other. A linear control strategy that achieves this objective is

$$
\dot{x}_{i}=\sum_{j \in \mathbb{N}_{i}}\left(x_{j}-x_{i}\right)
$$

as long as the graph $\mathrm{G}$ is connected for all times. However, as shown in [56], initially connected proximity networks that evolve according to (23) are not guaranteed to remain connected throughout time. Instead, nonlinear coordination models are needed, and one that achieves rendezvous while ensuring connectivity (Problem I) is the hybrid control strategy under consideration in Proposition 5.1. In particular, the model employed in [56] is

$$
\dot{x}_{i}=\sum_{j \in \mathbb{N}_{i}^{\sigma}} \frac{2 \rho_{2}^{2}}{\left(\rho_{2}^{2}-\left\|x_{i j}\right\|_{2}^{2}\right)^{2}}\left(x_{j}-x_{i}\right)
$$

which not only ensures that no edges are lost, but it also achieves rendezvous in the sense that all agents asymptotically approach the same location. This is due to the modified potentials $\bar{\psi}_{i j}=\left\|x_{i j}\right\|_{2}^{2} /\left(\rho_{2}^{2}-\left\|x_{i j}\right\|_{2}^{2}\right)$ in (24) that along with link maintenance (Section V-A) also capture the rendezvous objective. An example of this behavior is shown in Fig. 8.

It should be noted that the rendezvous control law often serves a cohesion purpose, i.e., ensures that the robots in the team stay close together. Nevertheless, exact rendezvous is not necessarily a good thing and a reactive, collision-avoidance controller could be added to the control strategy to avoid overlapping of the actual robots.

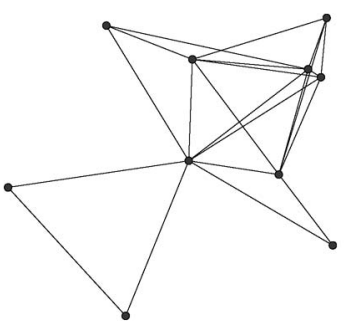

(a)

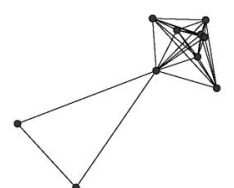

(b)

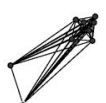

(c)
Fig. 8. Execution of the rendezvous control strategy until the graph is a complete graph.

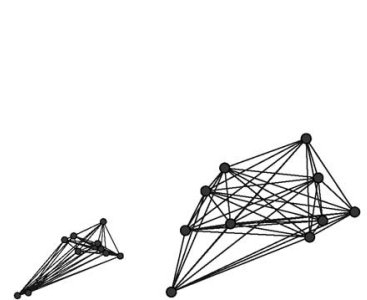

(a)

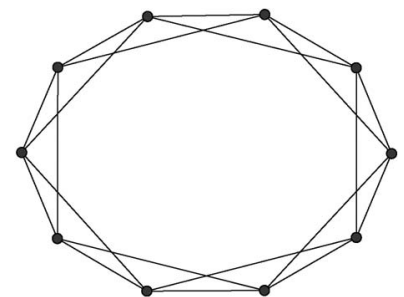

(c)
Fig. 9. Illustration of how the complete graph is changed to the desired formation using only local information.

\section{B. Connectivity Preserving Formation Control}

A variation to the rendezvous objective is the problem of driving the robots to a desired target configuration, rather than to a common target location. We assume that this target configuration can be encoded through $\zeta_{1}, \ldots$, $\zeta_{n} \in \mathbf{R}^{d}$, with the interpretation that agent $i$ should go to location $\zeta_{i}$, for $i=1, \ldots, n$. Since formations are considered rotationally and translationally invariant objects in the configuration space, their exact location is not of interest. Therefore, the formation control objective is to achieve

$$
x_{i}=\zeta_{i}+\tau \quad \forall i=1, \ldots, n
$$

for some constant $\tau \in \mathbf{R}^{d}$. In other words, $\tau$ corresponds to the constant offset from the target configuration that the agents should agree on. But, by letting $\chi_{i}=x_{i}-\zeta_{i}$, and

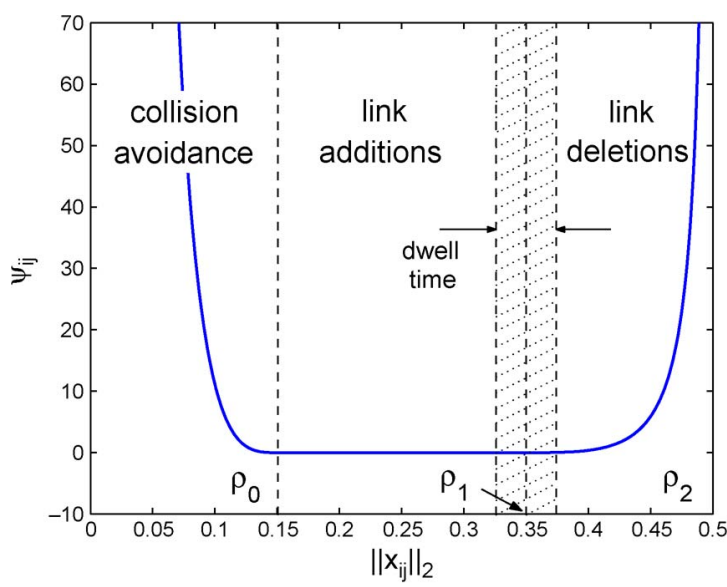

Fig. 10. The artificial potential function $\psi_{i j}\left(\left\|x_{i j}\right\|_{2}\right)$. The function is symmetric with respect to $x_{i}$ and $x_{j}$, and when bounded, it guarantees both collision avoidance for $\left\|x_{i j}\right\|_{2} \rightarrow \boldsymbol{O}$ and edge preservation for $\left\|x_{i j}\right\|_{2} \rightarrow \rho_{2}$. Here, the function is plotted for $\rho_{0}=0.15, \rho_{1}=0.35$, and $\rho_{2}=$ 0.5. The dwell time at the switching threshold $\rho_{2}$ ensures that the resulting switched system is well defined [64]. 


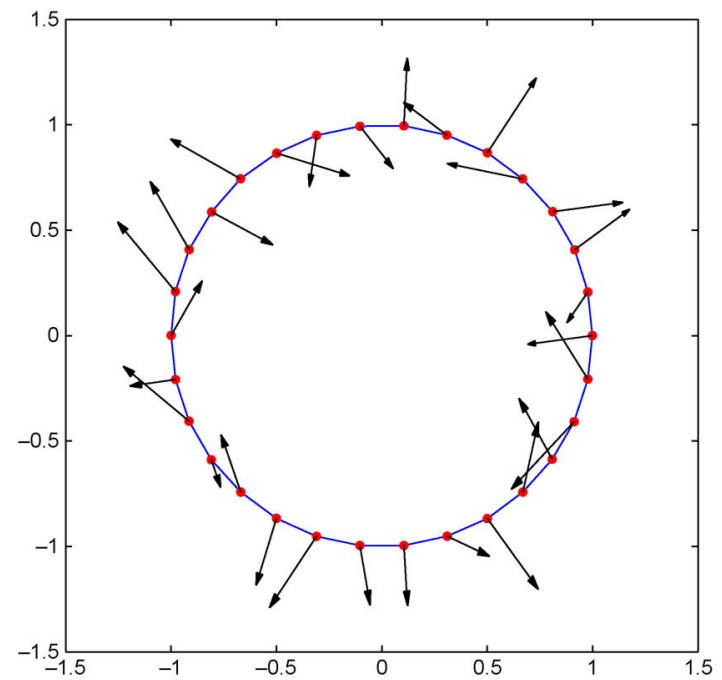

(a)

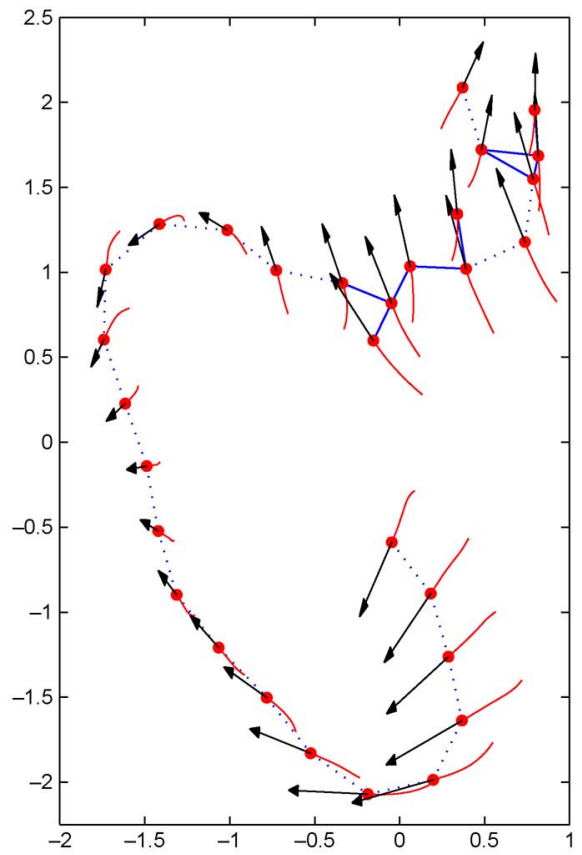

(c)

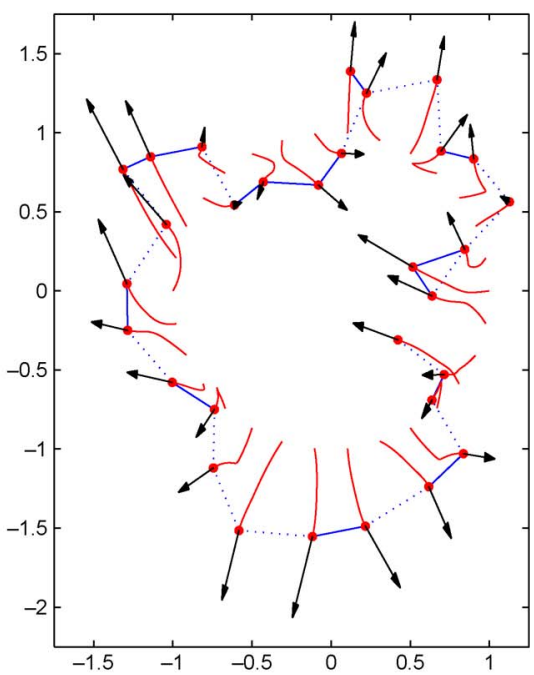

(b)

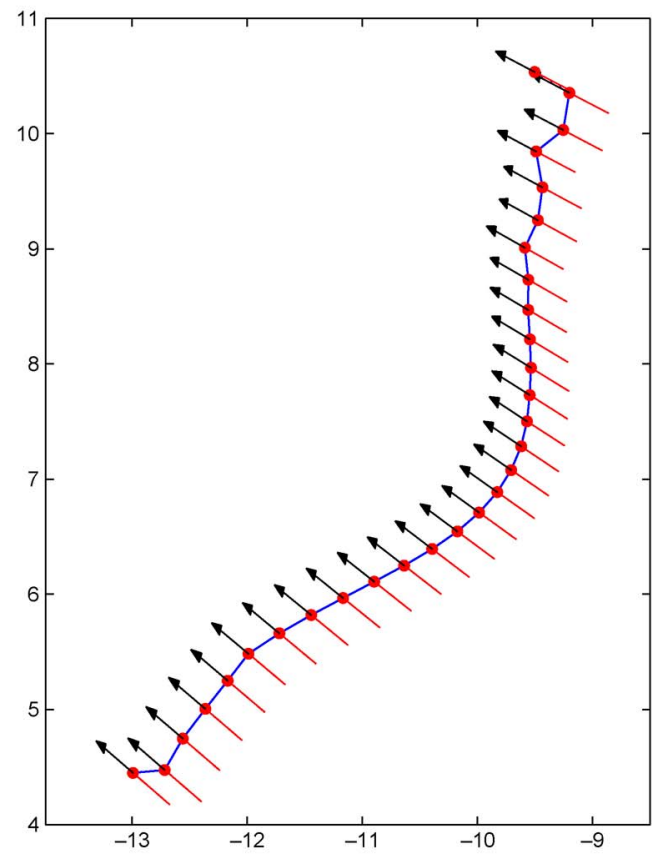

(d)

Fig. 11. Connectivity preserving flocking of $n=30$ robots for a sparse initial configuration where connectivity cannot be trivially maintained. It can be seen that the network remains connected while all robot velocities are asymptotically aligned. Dotted lines indicate communication links that are candidates for deletion (Fig. 10).

running the connectivity preserving rendezvous algorithm (24) over the $\chi_{i}$ 's instead of over the $x_{i}$ 's, it is ensured that the offsets $\chi_{i}$ reach a common value which corresponds directly to the offset $\tau$ [81]. Note that since $\dot{x}_{i}=\dot{\chi}_{i}$, this strategy directly gives desired motions for the robots in terms of their velocities. Moreover, all that is needed to compute these control laws are the relative displacements $x_{i}-x_{j}$ between neighboring robots as well as the desired predefined relative displacement $\zeta_{i}-\zeta_{j}$. This is highlighted in Fig. 9.

\section{Connectivity Preserving Flocking}

Flocking has been given many definitions and various models have been proposed so far [82]-[85]. Therefore, it is understood quite differently by different authors. In this paper, we focus on the model proposed by Reynolds, developed to simulate social aggregation phenomena, such as flocks of birds and schools of fish [86]. Reynolds called the generic simulated flocking creatures "boids" and developed his flocking model based on three simple steering behaviors that describe how an individual robot 
maneuvers given the positions and velocities of its nearby flockmates.

- Alignment: Steer towards the average heading of local flockmates.

- Separation: Steer to avoid crowding of local flockmates.

- Cohesion: Steer towards the average position of local flockmates.

In Reynold's model, every robot has access to the whole scene's geometric description, however, flocking requires information from nearest neighbor flockmates only. This neighborhood depends on a distance and an angle from the robot's direction of motion, and can be thought of as model of limited perception (such as fish in murky water) or as the region where a robot's motion is influenced by its flockmates. Superposition of these three rules results in all robots moving as a flock while avoiding collisions. Inspired by Reynold's model, Tanner et al. [87] proposed local control laws that allow a team of robots with double integrator dynamics

$$
\begin{aligned}
& \dot{x}_{i}=v_{i} \\
& \dot{v}_{i}=-\sum_{j \in \mathbb{N}_{i}}\left(v_{i}-v_{j}\right)-\sum_{j \in \mathbb{N}_{i}} \bar{\psi}_{i j}
\end{aligned}
$$

to align their velocities, move with a common speed, and achieve desired inter-robot distances while avoiding collisions with each other. Stability results were obtained using nonsmooth analysis and algebraic graph theory and critically relied on connectivity of the communication network. Based on these results, Zavlanos et al. [64] proposed integration of the dynamics (25) with the connectivity control framework developed in Section V-B and the artificial potentials (Fig. 10)

$$
\bar{\psi}_{i j}= \begin{cases}\frac{1}{\left\|x_{i j}\right\|_{2}^{2}}+P_{1}\left(\left\|x_{i j}\right\|_{2}\right), & \left\|x_{i j}\right\|_{2} \in\left(0, \rho_{0}\right] \\ 0, \frac{1}{\rho_{2}^{2}-\left\|x_{i j}\right\|_{2}^{2}}+P_{2}\left(\left\|x_{i j}\right\|_{2}\right), & \left\|x_{i j}\right\|_{2} \in\left(\rho_{0}, \rho_{1}\right) \\ & \| \rho_{i j} \in\left[\rho_{1}, \rho_{2}\right)\end{cases}
$$

with $0<\rho_{0}<\rho_{1}<\rho_{2}$ and $P_{k}\left(\left\|x_{i j}\right\|_{2}\right) \triangleq a_{k}\left\|x_{i j}\right\|_{2}^{2}+$ $b_{k}\left\|x_{i j}\right\|_{2}+c_{k}$ for $k=1,2$ such that $\psi_{i j} \in \mathbf{C}^{2}$ in $\left(0, \rho_{2}\right)$. The resulting multirobot hybrid system was shown to guarantee the flocking behavior of the team while preserving connectivity of the network (Fig. 11).

\section{CONCLUSION}

In this paper, we provided a theoretical framework for controlling graph connectivity in mobile robot networks. We presented a cohesive overview of the key results in [41]-[43], [46], and [56] and discussed basic notions of network connectivity as well as control-theoretic methods for connectivity preservation. These methods relied on a variety of mathematical tools, ranging from spectral graph theory and semidefinite programming to maximize the algebraic connectivity of a network, to gradient-descent algorithms and hybrid systems to ensure topology control in a least restrictive manner. We also discussed applications of connectivity control to multirobot rendezvous [56], flocking [64], and formation control [56], where so far, network connectivity had been considered an assumption. A byproduct of this work was to classify the available literature with respect to the connectivity metrics and solution techniques and provide a reference for future research.

\section{REFERENCES}

[1] J. Cortes, "Achieving coordination tasks in finite time via nonsmooth gradient flows," in Proc. 44th IEEE Conf. Decision Control/Eur. Control Conf., Seville, Spain, Dec. 2005, pp. 6376-6381.

[2] J. Cortes, "Distributed algorithms for reaching consensus on general functions," Automatica, vol. 44, no. 3, pp. 726-737, 2008.

[3] R. Olfati-Saber and R. M. Murray, "Decentralized control of connectivity for multi-agent systems," in Proc. Amer. Control Conf., 2003, pp. 951-956.

[4] R. Olfati-Saber and R. M. Murray, "Consensus problems in networks of agents with switching topology and time-delays," IEEE Trans. Autom. Control, vol. 49, no. 9, pp. 1520-1533, Sep. 2004

[5] J. A. Fax and R. M. Murray, "Information flow and cooperative control of vehicle formations," IEEE Trans. Autom. Control, vol. 49, no. 9, pp. 1465-1476, Sep. 2004.

[6] A. Jadbabaie, J. Lin, and A. S. Morse, "Coordination of groups of mobile autonomous agents using nearest neighbor rules," IEEE Trans. Autom. Control, vol. 48 no. 6, pp. 988-1001, Jun. 2003.
[7] L. Moreau, "Stability of multi-agent systems with time-dependent communication links," IEEE Trans. Autom. Control, vol. 50, no. 2, pp. 169-182, Feb. 2005.

[8] W. Ren and R. W. Beard, "Consensus seeking in multi-agent systems under dynamically changing interaction topologies," IEEE Trans. Autom. Control, vol. 50, no. 5, pp. 655-661, May 2005.

[9] Z. Lin, M. Brouke, and B. Francis, "Local control strategies for groups of mobile autonomous agents," IEEE Trans. Autom. Control, vol. 49, no. 4, pp. 622-629, Apr. 2004

[10] V. Blondel, J. M. Hendrickx, A. Olshevsky, and J. N. Tsitsiklis, "Convergence in multi-agent coordination, consensus, and flocking," in Proc. 44th IEEE Conf. Decision Control/Eur. Control Conf., Seville, Spain, Dec. 2005, pp. 2996-3000.

[11] D. Bauso, L. Giarre, and R. Pesenti, "Nonlinear protocols for optimal distributed consensus in networks of dynamic agents," Syst. Control Lett., vol. 55, no. 11, pp. 918-928, 2006.

[12] M. Mehyar, D. Spanos, J. Pongsjapan, S. H. Low, and R. M. Murray, "Achieving coordination tasks in finite time via nonsmooth gradient flows," in Proc. 44th IEEE Conf. Decision Control/Eur. Control Conf., Seville, Spain, Dec. 2005, pp. 7446-7451.

[13] P.-A. Bliman and G. Ferrari-Trecate, "Average consensus problems in networks of agents with delayed communications," in Proc. 44th IEEE Conf. Decision Control/Eur. Control Conf., Seville, Spain, Dec. 2005, pp. 7066-7071.

[14] R. Olfati-Saber, J. A. Fax, and R. M. Murray, "Consensus and cooperation in networked multi-agent systems," Proc. IEEE, vol. 95, no. 1, pp. 215-233, Jan. 2007.

[15] A. Neskovic, N. Neskovic, and G. Paunovic, "Modern approaches in modeling of mobile radio systems propagation environment," IEEE Commun. Surv., vol. 3, no. 3, pp. 1-12, 2000

[16] J. D. Parsons, The Mobile Radio Propagation Channel. New York: Wiley, 2000.

[17] K. Pahlavan and A. H. Levesque, Wireless Information Networks. New York: Wiley, 1995.

[18] A. J. Goldsmith and S. B. Wicker, "Design challenges for energy-constrained ad hoc 
wireless networks," IEEE Wireless Commun. Mag., vol. 9, no. 4, pp. 8-27, Aug. 2002.

[19] P. Gupta and P. R. Kumar, "Critical power for asymptotic connectivity," in Proc. 37th IEEE Conf. Decision Control, Tampa, FL, Dec. 1998, pp. 1106-1110.

[20] B. Chen, K. Jamieson, H. Balakrishnan, and R. Morris, "Span: An energy-efficient coordination algorithm for topology maintenance in ad hoc wireless networks," Wireless Netw., vol. 8, no. 5, pp. 481-494, Sep. 2002.

[21] R. Ramanathan and R. Rosales-Hain, "Topology control of multihop wireless networks using transmit poweradjustment," in Proc. 19th Annu. Joint Conf. IEEE Comput. Commun. Soc. (INFOCOM), Tel Aviv, Israel, 2000, pp. 404-413.

[22] E. L. Lloyd, R. Liu, M. V. Marathe R. Ramanathan, and S. S. Ravi, "Algorithmic aspects of topology control problems for ad hoc networks," Mobile Netw. Appl., vol. 10, no. 1-2, pp. 19-34, Feb. 2005.

[23] L. Ning, J. C. Hou, and L. Sha, "Design and analysis of an mst-based topology control algorithm," IEEE Trans. Wireless Commun., vol. 4, no. 3, pp. 1195-1206, May 2005.

[24] R. D'Souza, D. Galvin, C. Moore, and D. Randall, "Global connectivity from local geometric constraints for sensor networks with various wireless footprints," in Proc. 5th Int. Conf. Inf. Process. Sensor Netw., Nashville, TN, Apr. 2006, pp. 19-26.

[25] L. Li, J. Y. Halpern, P. Bahl, Y. M. Wang, and R. Wattenhofer, "A cone-based distributed topology-control algorithm for wireless multi-hop networks," IEEE/ACM Trans. Netw., vol. 13, no. 1, pp. 147-159, Feb. 2005.

[26] S. Poduri, S. Pattem, B. Krishnamachari, and G. S. Sukhatme, "Using local geometry for tunable topology control in sensor networks," IEEE Trans. Mobile Comput., vol. 8, no. 2, pp. 218-230, Feb. 2009.

[27] R. Wattenhofer and A. Zollinger, "Xtc: A practical topology control algorithm for ad-hoc networks," in Proc. 18th IEEE Int. Parallel Distrib. Process. Symp., Santa Fe, NM, Apr. 2004, pp. 216-223.

[28] M. M. Zavlanos, A. Tahbaz-Salehi, A. Jadbabaie, and G. J. Pappas, "Distributed topology control of dynamic networks," in Proc. Amer. Control Conf., Seattle, WA, Jun. 2008, pp. 2660-2665.

[29] F. Xue and P. R. Kumar, "The number of neighbors needed for connectivity of wireless networks," Wireless Netw., vol. 10, no. 2, pp. 169-181, Mar. 2004.

[30] O. Dousse, F. Baccelli, and P. Thiran, "Impact of interferences on connectivity in ad hoc networks," IEEE/ACM Trans. Netw., vol. 13, no. 2, pp. 425-436, 2005.

[31] M. Fiedler, "Absolute algebraic connectivity of trees," Linear Multilinear Algebra, vol. 26, pp. 85-106, 1990.

[32] Y. Wan, S. Roy, X. Wang, A. Saberi, T. Yang, M. Xue, and B. Malek, "On the structure of graph edge designs that optimize the algebraic connectivity," in Proc. 47th IEEE Conf. Decision Control, Cancun, Mexico, Dec. 2008, pp. 805-810.

[33] A. Ghosh and S. Boyd, "Growing well-connected graphs," in Proc. 45th IEEE Conf. Decision Control, San Diego, CA, Dec. 2006, pp. 6605-6611.

[34] S. Boyd, "Convex optimization of graph Laplacian eigenvalues," in Proc. Int. Congr. Math., Madrid, Spain, Aug. 2006, pp. 1311-1319.
[35] M. Mesbahi, "On state-dependent dynamic graphs and their controllability properties," IEEE Trans. Autom. Control, vol. 50, no. 3, pp. 387-392, Mar. 2005.

[36] H. Ando, Y. Oasa, I. Suzuki, and M. Yamashita, "Distributed memoryless point convergence algorithm for mobile robots with limited visibility," IEEE Trans. Robot. Autom., vol. 15, no. 5, pp. 818-828, Oct. 1999.

[37] M. Fiedler, "Algebraic connectivity of graphs," Czechoslovak Math. J., vol. 23, no. 98, pp. 298-305, 1973.

[38] B. Mohar, "The Laplacian spectrum of graphs," in Graph Theory, Combinatorics, and Applications, Y. Alavi, G. Chartrand, O. Ollermann, and A. Schwenk, Eds. New York: Wiley, 1991, pp. 871-898.

[39] R. Merris, "Laplacian matrices of a graph: A survey," Linear Algebra Its Appl., vol. 197, pp. 143-176, 1994.

[40] C. Godsil and G. Royle, Algebraic Graph Theory, vol. 207. Berlin, Germany: Springer-Verlag, 2001, ser. Graduate Texts in Mathematics.

[41] Y. Kim and M. Mesbahi, "On maximizing the second smallest eigenvalue of a state-dependent graph Laplacian," IEEE Trans. Autom. Control, vol. 51, no. 1, pp. 116-120, Jan. 2006.

[42] M. C. DeGennaro and A. Jadbabaie, "Decentralized control of connectivity for multi-agent systems," in Proc. 45th IEEE Conf. Decision Control, San Diego, CA, Dec. 2006, pp. 3628-3633.

[43] M. M. Zavlanos and G. J. Pappas, "Potential fields for maintaining connectivity of mobile networks," IEEE Trans. Robot., vol. 23, no. 4, pp. 812-816, Aug. 2007.

[44] P. Yang, R. Freeman, G. Gordon, K. Lynch, S. Srinivasa, and R. Sukthankar, "Decentralized estimation and control of graph connectivity in mobile sensor networks," in Proc. Amer. Control Conf., Seattle, WA, Jun. 2008, pp. 2678-2683.

[45] F. Knorn, R. Stanojevic, M. Corless, and R. Shorten, "A framework for decentralized feedback connectivity control with application to sensor networks," Int. J. Control, vol. 82, no. 11, pp. 2095-2114, 2009.

[46] M. M. Zavlanos and G. J. Pappas, "Distributed connectivity control of mobile networks," IEEE Trans. Robot., vol. 24, no. 6, pp. 1416-1428, Dec. 2008.

[47] M. M. Zavlanos and G. J. Pappas, "Controlling connectivity of dynamic graphs," in Proc. 44th IEEE Conf. Decision Control/Eur. Control Conf., Seville, Spain, Dec. 2005, pp. 6388-6393.

[48] K. Srivastava and M. W. Spong, "Multi-agent coordination under connectivity constraints," in Proc. Amer. Control Conf., Seattle, WA, Jun. 2008, pp. 2648-2653.

[49] G. Notarstefano, K. Savla, F. Bullo, and A. Jadbabaie, "Maintaining limited-range connectivity among second-order agents," in Proc. Amer. Control Conf., Minneapolis, MN, Jun. 2006, pp. 2124-2129.

[50] F. Bullo, J. Cortes, and S. Martinez, Distributed Control of Robotic Networks. Princeton, NJ: Princeton Univ. Press, 2009, ser. Distributed Control of Robotic Networks.

[51] D. P. Spanos and R. M. Murray, "Robust connectivity of networked vehicles," in Proc. 43rd IEEE Conf. Decision Control, Bahamas, Dec. 2004, pp. 2893-2898.
[52] D. V. Dimarogonas and K. J. Kyriakopoulos, "Connectedness preserving distributed swarm aggregation for multiple kinematic robots," IEEE Trans. Robot., vol. 24, no. 5, pp. 1213-1223, Oct. 2008

[53] A. Cornejo and N. Lynch, "Connectivity service for mobile ad-hoc networks," in Proc. 2nd IEEE Int. Conf. Self-Adaptive Self-Organizing Syst. Workshops, Oct. 2008, pp. 292-297.

[54] Z. Yao and K. Gupta, "Backbone-based connectivity control for mobile networks," in Proc. IEEE Int. Conf. Robot. Autom., Kobe, Japan, May 2009, pp. 1133-1139.

[55] M. M. Zavlanos, A. Jadbabaie, and G. J. Pappas, "Flocking while preserving network connectivity," in Proc. 46th IEEE Conf. Decision Control, New Orleans, LA, Dec. 2007, pp. 2919-2924.

[56] M. Ji and M. Egerstedt, "Coordination control of multi-agent systems while preserving connectedness," IEEE Trans. Robot., vol. 23, no. 4, pp. 693-703, Aug. 2007.

[57] J. Wagenpfeil, A. Trachte, T. Hatanaka, M. Fujita, and O. Sawodny, "A distributed minimum restrictive connectivity maintenance algorithm," in Proc. 9th Int. Symp. Robot Control, Gifu, Japan, pp. 499-504, Sep. 2009.

[58] M. Schuresko and J. Cortes, "Distributed motion constraints for algebraic connectivity of robotic networks," J. Intell. Robot. Syst., vol. 56, no. 1-2, pp. 99-126, Sep. 2009.

[59] M. Schuresko and J. Cortes, "Distributed tree rearrangements for reachability and robust connectivity," in Hybrid Systems: Computetation and Control, vol. 5469, Berlin, Germany: Springer-Verlag, 2009, pp. $470-474$.

[60] D. P. Spanos and R. M. Murray, "Motion planning with wireless network constraints," in Proc. Amer. Control Conf., Portland, OR, Jun. 2005, pp. 87-92.

[61] E. Stump, A. Jadbabaie, and V. Kumar, "Connectivity management in mobile robot teams," in Proc. IEEE Int. Conf. Robot. Autom., Pasadena, CA, May 2008, pp. $1525-1530$.

[62] A. Ganguli, J. Cortes, and F. Bullo, "Multirobot rendezvous with visibility sensors in nonconvex environments," IEEE Trans. Robot., vol. 25, no. 2, pp. 340-352, Apr. 2009

[63] J. Cortes, S. Martinez, and F. Bullo, "Robust rendezvous for mobile autonomous agents via proximity graphs in arbitrary dimensions," IEEE Trans. Autom. Control, vol. 51, no. 8 , pp. 1289-1298, Aug. 2006

[64] M. M. Zavlanos, H. G. Tanner, A. Jadbabaie, and G. J. Pappas, "Hybrid control for connectivity preserving flocking," IEEE Trans. Autom. Control, vol. 54, no. 12, pp. 2869-2875, Dec. 2009.

[65] D. V. Dimarogonas and K. H. Johansson, "Decentralized connectivity maintenance in mobile networks with bounded inputs," in Proc. IEEE Int. Conf. Robot. Autom., Pasadena, CA, May 2008, pp. 1507-1512.

[66] A. Ajorlou and A. G. Aghdam, "A class of bounded distributed controllers for connectivity preservation of unicycles," in Proc. 49th IEEE Conf. Decision Control, Atlanta, GA, Dec. 2010, pp. 3072-3077.

[67] A. Ajorlou, A. Momeni, and A. G. Aghdam, "A class of bounded distributed control strategies for connectivity preservation in multi-agent systems," IEEE Trans. Autom. Control, vol. 55, no. 12, pp. 2828-2833, Dec. 2010. 
[68] M. A. Hsieh, A. Cowley, V. Kumar, and C. Taylor, "Maintaining network connectivity and performance in robot teams," J. Field Robot., vol. 25, no. 1-2, pp. 111-131, 2008.

[69] A. R. Wagner and R. C. Arkin, "Communication-sensitive multi-robot reconnaissance," in Proc. IEEE Int. Conf. Robot. Autom., New Orleans, LA, 2004, pp. 2480-2487.

[70] M. Powers and T. Balch, "Value-based communication preservation for mobile robots," in Distrib. Autonom. Robot. Syst. 6 , R. Alami, R. Chatila, and H. Asama, Eds. Japan: Springer, 2007, pp. 327-336.

[71] Y. Mostofi, "Decentralized communication-aware motion planning in mobile networks: An information-gain approach," J. Intell. Robot. Syst., vol. 56, no. 1-2, pp. 233-256, 2009.

[72] P. Flocchini, G. Prencipe, N. Santoro, and P. Widmayer, "Gathering of asynchronous oblivious robots with limited visibility," Theor. Comput. Sci., vol. 337, no. 1-3, pp. 147-168, 2005.

[73] R. C. Arkin and J. Diaz, "Line-of-sight constrained exploration for reactive multiagent robotic teams," in Proc. 7th Int Workshop Adv. Motion Control, Maribor, Slovenia, 2002, pp. 455-461.
[74] S. O. Anderson, R. Simmons, and D. Goldberg, "Maintaining line-of-sight communications networks between planetray rovers," in Proc. IEEE/RSJ Int. Conf. Intell. Robots Syst., Las Vegas, NV, 2003, pp. 2266-2272.

[75] G. Hollinger and S. Singh, "Multi-robot coordination with periodic connectivity," in Proc. IEEE Int. Conf. Robot. Autom., 2010.

[76] T. Gustavi, D. V. Dimarogonas, M. Egerstedt, and $\mathrm{X}$. Hu, "Sufficient conditions for connectivity maintenance and rendezvous in leader-follower networks," Automatica, vol. 46, no. 1, pp. 133-139, Jan. 2010.

[77] D. Tardioli, A. R. Mosteo, L. Riazuelo, J. L. Villarroel, and L. Montano, "Enforcing network connectivity in robot team missions," Int. J. Robot. Res., to be published.

[78] N. Michael, M. M. Zavlanos, V. Kumar, and G. J. Pappas, "Maintaining connectivity in mobile robot networks," in Experimental Robotics, New York: Springer-Verlag, 2009, pp. 117-126.

[79] S. Boyd and L. Vandenberghe, Convex Optimization. Cambridge, U.K.: Cambridge Univ. Press, 2004.

[80] D. Kempe and M. McSherry, "A decentralized algorithm for spectral analysis," J. Comput. Syst. Sci., vol. 74, no. 1, pp. 70-83, 2008.
[81] M. Mesbahi and M. Egerstedt, Graph Theoretic Methods for Multiagent Networks. Princeton, NJ: Princeton Univ. Press, 2010.

[82] A. Barbosa, "The effects of predation risk on scanning and flocking behavior in dunlin," J. Field Ornithology, vol. 68, no. 4, pp. 607-612, 1997.

[83] N. Leonard and E. Fiorelli, "Virtual leaders, artificial potentials and coordinated control of groups," in Proc. 40th IEEE Conf. Decision Control, Orlando, FL, Dec. 2001 pp. 2968-2973.

[84] R. O. Saber and R. M. Murray, "Flocking with obstacle avoidance: Cooperation with limited communication in mobile networks," in Proc. 42nd IEEE Conf. Decision Control, Maui, HI, Dec. 2003, pp. 2022-2028.

[85] J. Toner and Y. Tu, "Flocks, herds and schools: A quantitative theory of flocking," Phys. Rev. E, vol. 58, no. 4, pp. 4828-4858, Oct. 1998.

[86] C. Reynolds, "Flocks, birds and schools: A distributed behavioral model," ACM SIGGRAPH Comput. Graph., vol. 21, no. 4, pp. 25-34, 1987.

[87] H. G. Tanner, A. Jadbabaie, and G. J. Pappas, "Flocking in fixed and switching networks," IEEE Trans. Autom. Control, vol. 52, no. 5, pp. 863-868, May 2007.

\section{ABOUT THE AUTHORS}

Michael M. Zavlanos (Member, IEEE) received the Diploma in mechanical engineering from the National Technical University of Athens, Athens, Greece, in 2002, and the M.S.E. and Ph.D. degrees in electrical and systems engineering from the University of Pennsylvania, Philadelphia, in 2005 and 2008, respectively.

He is currently an Assistant Professor with the Department of Mechanical Engineering, Stevens Institute of Technology, Hoboken, NJ. His current research interests include a wide range of topics in the emerging discipline of networked systems and science, with applications in robotic, sensor, biomolecular, and social networks. He is particularly interested in hybrid solution techniques, on the interface of control theory with the discrete science of networks and graphs.

Dr. Zavlanos was a recipient of the National Science Foundation (NSF) Faculty Early Career Development (CAREER) Award in 2011 and a finalist for the Best Student Paper Award at the 45th IEEE Conference on Decision and Control in 2006.

Magnus B. Egerstedt (Senior Member, IEEE) received the B.A. degree in philosophy from Stockholm University, Stockholm, Sweden, in 1996 and the M.S. degree in engineering physics and the Ph.D. degree in applied mathematics from the Royal Institute of Technology, Stockholm, Sweden, in 1996 and 2000, respectively.

$\mathrm{He}$ is a Professor in the School of Electrical and Computer Engineering, Georgia Institute of Technology, Atlanta, where he has been on the faculty since 2001 . His research interests include hybrid and networked control,

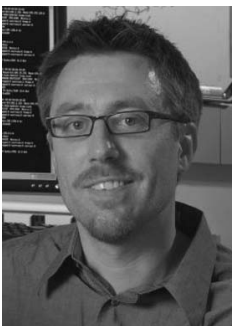

with applications in motion planning, control, and coordination of mobile robots.

Dr. Egerstedt serves as Editor for Electronic Publications for the IEEE Control Systems Society and Associate Editor for the IEEE TRANSACTIONS on Automatic Control. He is the Director of the Georgia Robotics and Intelligent Systems Laboratory (GRITS Lab). He received the ECE/GT Outstanding Junior Faculty Member Award in 2005 and the CAREER award from the U.S. National Science Foundation in 2003.

George J. Pappas (Fellow, IEEE) received the Ph.D. degree in electrical engineering and computer sciences from the University of California at Berkeley, Berkeley, in 1998, where he received the Eliahu Jury Award for Excellence in Systems Research.

$\mathrm{He}$ is currently the Joseph Moore Professor of Electrical and Systems Engineering at the University of Pennsylvania, Philadelphia. He is a member of the General Robotics, Automation, Sensing and

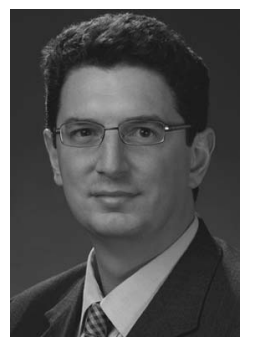
Perception (GRASP) Laboratory and serves as the Deputy Dean for Research in the School of Engineering and Applied Science. His current research interests include hybrid and embedded systems, hierarchical control systems, distributed control systems, nonlinear control systems, with applications to robotics, unmanned aerial vehicles, biomolecular networks, and green buildings.

Prof. Pappas has received numerous awards, including the National Science Foundation (NSF) CAREER Award in 2002, the NSF Presidential Early Career Award for Scientists and Engineers in 2002, the 2009 George S. Axelby Outstanding Paper Award, and the 2010 Anotnio Ruberti Outstanding Young Researcher Prize. 